\title{
Pricing Vulnerable Options with Market Prices of Common Jump Risks under Regime-Switching Models
}

\author{
Miao Han $\mathbb{D}^{1},{ }^{1}$ Xuefeng Song, ${ }^{2}$ Huawei Niu, ${ }^{3}$ and Shengwu Zhou $\mathbb{D}^{1}$ \\ ${ }^{1}$ School of Mathematics, China University of Mining and Technology, Xuzhou, China \\ ${ }^{2}$ School of Management Science and Engineering, Nanjing University of Finance and Economics, Nanjing, China \\ ${ }^{3}$ School of Finance and Center for Financial Engineering, Nanjing Audit University, Nanjing, China \\ Correspondence should be addressed to Miao Han; luckhanmiao@126.com
}

Received 2 September 2017; Accepted 14 December 2017; Published 21 January 2018

Academic Editor: Paolo Renna

Copyright (C) 2018 Miao Han et al. This is an open access article distributed under the Creative Commons Attribution License, which permits unrestricted use, distribution, and reproduction in any medium, provided the original work is properly cited.

\begin{abstract}
This paper investigates the valuation of vulnerable European options considering the market prices of common systematic jump risks under regime-switching jump-diffusion models. The way of regime-switching Esscher transform is adopted to identify an equivalent martingale measure for pricing vulnerable European options. Explicit analytical pricing formulae for vulnerable European options are derived by risk-neutral pricing theory. For comparison, the other two cases are also considered separately. The first case considers all jump risks as unsystematic risks while the second one assumes all jumps risks to be systematic risks. Numerical examples for the valuation of vulnerable European options are provided to illustrate our results and indicate the influence of the market prices of jump risks on the valuation of vulnerable European options.
\end{abstract}

\section{Introduction}

Along with the development of the OTC market, people have recognized the influence of credit risk on financial derivatives pricing and attempted to establish all kinds of credit risk models. Johnson and Stulz [1] first incorporate credit risk with option pricing models and call it vulnerable options, which are vulnerable to counterparty default. They assume that if the counterparty writing an option is unable to make a promised payment, the holder of a derivative security would receive all assets of the counterparty. Klein [2] extends the work of Johnson and Stulz [1] by allowing the option writer to have other liabilities, which rank equally with payments under the option. It also extends the work of Hull and White [3] by relaxing the assumption of independence between the assets of the counterparty and the asset underlying the option. Jarrow and Turnbull [4] provide a new methodology for pricing and hedging derivative securities involving two types of credit risks. Klein and Inglis [5] also expand on the results of pricing vulnerable European options when the payoff of the option can increase the risk of financial distress from Johnson and Stulz [1] and Klein [2]. Hung and Liu [6] extend the framework of Klein [2] to price vulnerable option pricing when the market is incomplete. Chang and Huang [7] and Klein and Yang [8] study the valuation of vulnerable American options with correlated credit risk.

However, the above literatures on vulnerable options all assume that the dynamics of the assets are modelled by geometric Brownian motions with constant drift and volatility. Indeed, over the past decade or two, dozens of empirical evidences have revealed that risky asset prices not only present sudden shocks due to the arrival of important new information in financial markets but also exhibit different behaviors in different time periods due to the time-inhomogeneity generated by the financial market. For the former case, Merton [9] introduces the jump-diffusion models with compound Poisson processes into option pricing (see Kou [10], Xu et al. [11], Tian et al. [12], etc.). For the latter case, Markov regime-switching models have provided us with a natural and convenient way to describe structural changes in market interest rate, exchange rate, stock returns, and so forth, since Hamilton [13] introduced this class of models into economics and finance. 
To better describe both the time-inhomogeneity and sudden shocks in the processes of asset prices, there have been lots of papers studying options pricing through incorporating jump-diffusion models with Markov regime-switching models. Elliott and Osakwe [14] consider option pricing for pure jump processes with Markov switching compensators. Bo et al. [15] study the pricing of some currency options based on the Markov-modulated jump-diffusion models for the spot foreign exchange rate. Wang and Wang [16] and Niu and Wang [17] study the pricing problem of vulnerable European options under the Markov regime switching jump-diffusion models. However, they regard all jump risks as systematic risks. Indeed, the jumps in an actual process may be caused by the market or may occur on its own. Tian et al. [12] divide the jumps into individual jumps for each asset price and common jumps that affect the prices of all assets. In this paper, considering the market prices of common systematic jump risks regardless of individual jump risks, we develop an equivalent martingale measure for two regime-switching jump-diffusion processes with correlated jumps via regimeswitching Esscher transform and consider the differences between the physical jump-diffusion processes and the riskneutral jump-diffusion processes. The dynamics of the risk assets prices in this paper are different from those obtained by considering the market prices of all jump risks in Niu and Wang [17] under the risk-neutral measure. In order to consider the influence of the market prices of jump risks on vulnerable option values, we also study vulnerable options pricing formulae with or without market prices of all jump risks. Finally, numerical results are also presented to illustrate our results by the Monte Carlo simulations.

The rest of the paper is organized as follows. Section 2 presents the basic setting and asset price model. In Section 3, we employ the regime-switching Esscher transform to determine an equivalent martingale measure considering the market prices of common systematic jump risks and derive the closed-form pricing formulae for vulnerable European options under a Markov regime switching jump-diffusion model. In Section 4, for comparison, the pricing formulae for vulnerable European options are obtained taking the market prices of all jump risks into consideration. Section 5 presents some numerical results to illustrate the valuation of vulnerable options for different parameters and different cases. Section 6 concludes the paper.

\section{The Model Description}

Consider a continuous-time financial market with a finite time horizon $\mathscr{T}:=[0, T]$, where $T<\infty$. We consider a complete probability space $(\Omega, \mathscr{F}, P)$, where $P$ is a physical probability measure, under which all stochastic processes are defined. We equip the probability space $(\Omega, \mathscr{F}, P)$ with a filtration $\mathscr{F}:=\left\{\mathscr{F}_{t} \mid t \in \mathscr{T}\right\}$ and assume that there are three primary securities, namely, a risk-free bond $B$, underlying asset $S$, and option writer's asset $V$, which are traded continuously over the time horizon $\mathscr{T}:=[0, T] . U=$ $\left\{U_{t}\right\}_{t \in \mathscr{T}}$ is a continuous-time finite-state Markov chain on $(\Omega, \mathscr{F}, P)$ with a finite-state space $\mathcal{S}:=\left(s_{1}, s_{2}, \ldots, s_{N}\right)$. We use the states of $U$ to indicate the states of the economy. We adopt the assumptions of Elliott et al. [18] that the state space of $U$ is a limited collection of vectors $\left\{e_{1}, e_{2}, \ldots, e_{N}\right\}$, where $e_{i}=(0, \ldots, 1, \ldots, 0)^{\prime} \in \mathbf{R}^{N}$ with "1" in the $i$ th component. Suppose that the time-invariant generator matrix of $U$ is $A$ with $\left(a_{i j}\right)_{i, j=1,2, \ldots, N}$. Then, following Elliott et al. [18], the semimartingale decomposition of $U$ is given by

$$
d U_{t}=A U_{s} d s+d M_{t}
$$

where $M=\left\{M_{t}\right\}_{t \in \mathscr{T}}$ is an $\mathbf{R}^{N}$-valued martingale with respect to the filtration generated by $\left\{U_{t}\right\}_{t \in \mathscr{T}}$ under $P$.

Let $B_{t}$ denote the value of the bank currency account at time $t$. If one initially saves $B_{0}=1$, then he can gain $B_{t}=\exp \left(\int_{0}^{t} r_{s} d s\right)$ at time $t$. We assume that the instantaneous market interest rates $r_{t}$ depend on the economic states of $U$. Then, $r_{t}$ is given by

$$
r_{t}=\left\langle\mathbf{r}, U_{t}\right\rangle
$$

where $\mathbf{r}=\left(r_{1}, r_{2}, \ldots, r_{N}\right) \in \mathbf{R}^{N}$ with $r_{i}>0$, for each $i=$ $1,2, \ldots, N ;\langle\cdot, \cdot\rangle$ denotes the inner product in $\mathbf{R}^{N}$. For $i \neq j$, $\left\langle e_{i}, e_{j}\right\rangle=0$, else, $\left\langle e_{i}, e_{i}\right\rangle=1$.

Let the dynamics of the underlying asset value $S_{t}$ of the option follow a regime-switching jump-diffusion process under the physical measure $P$,

$$
\frac{d S_{t}}{S_{t-}}=\left(\alpha_{t}-k_{1} \lambda_{S t}^{*}\right) d t+\sigma_{S t} d W_{t}^{(1)}+\left(e^{Z_{S t-}^{(1)}}-1\right) d M_{t}^{(1)},
$$

where $W_{t}^{(1)}$ is a standard Brownian motion on $(\Omega, \mathscr{F}, P)$. The expected return rate $\left(\alpha_{t}\right)_{t \in \mathscr{T}}$ and the volatility $\left(\sigma_{S t}\right)_{t \in \mathscr{T}}$ of the underlying asset $S_{t}$ depend on $\left\{U_{t}\right\}_{t \in \mathscr{T}}$. They are defined as

$$
\begin{gathered}
\alpha_{t}=\left\langle\alpha, U_{t}\right\rangle, \\
\sigma_{S t}=\left\langle\sigma_{S}, U_{t}\right\rangle,
\end{gathered}
$$

where $\alpha=\left(\alpha_{1}, \alpha_{2}, \ldots, \alpha_{N}\right) \in \mathbf{R}^{N}$ and $\sigma_{S}=\left(\sigma_{S 1}, \sigma_{S 2}, \ldots\right.$, $\left.\sigma_{S N}\right) \in \mathbf{R}^{N}$ with $\sigma_{S i}>0$ for each $i=1,2, \ldots, N . M_{t}^{(1)}$ is a Poisson process with intensity $\lambda_{S t}^{*}$. Following Tian et al. [12], both the jump term $M_{t}^{(1)}$ and the intensity $\lambda_{S t}^{*}$ consist of two parts,

$$
\begin{gathered}
M_{t}^{(1)}=N_{t}^{(1)}+N_{t}, \\
\lambda_{S t}^{*}=\lambda_{S t}+\lambda_{t},
\end{gathered}
$$

where, specifically, shocks to the underlying asset price are also composed of two parts: individual shocks $N_{t}^{(1)}$ for each asset price and common shocks $N_{t}$ affecting the prices of all assets. Here, we take the common shocks as market factors, which are considered as systematic risks and consider the individual shocks as unsystematic risks. We assume $N_{t}^{(1)}$ and $N_{t}$ are independent Poisson processes with intensities $\lambda_{S t}$ and $\lambda_{t}$, which depend on the states of $\left\{U_{t}\right\}_{t \in \mathscr{T}}$ and are described by

$$
\begin{aligned}
& \lambda_{S t}^{*}=\left\langle\lambda_{S}^{*}, U_{t}\right\rangle, \\
& \lambda_{S t}=\left\langle\lambda_{S}, U_{t}\right\rangle, \\
& \lambda_{t}=\left\langle\lambda, U_{t}\right\rangle,
\end{aligned}
$$


where $\lambda_{S}^{*}=\left(\lambda_{S 1}^{*}, \lambda_{S 2}^{*}, \ldots, \lambda_{S N}^{*}\right) \in \mathbf{R}^{N}, \lambda_{S}=\left(\lambda_{S 1}, \lambda_{S 2}, \ldots\right.$, $\left.\lambda_{S N}\right) \in \mathbf{R}^{N}$, and $\lambda=\left(\lambda_{1}, \lambda_{2}, \ldots, \lambda_{N}\right) \in \mathbf{R}^{N}$. If the jump occurs at time $t$, the jump amplitude of the underlying asset is controlled by $Z_{S t}^{(1)}$. For any time $t \neq s$, we assume that $Z_{S t}^{(1)}$ and $Z_{S s}^{(1)}$ are independently and identically distributed and

$$
Z_{S t}^{(1)} \sim N\left(\mu_{1}, \sigma_{1}^{2}\right)
$$

The mean percentage jump size of the price is given by

$$
k_{1}=E\left(e^{Z_{S t}^{(1)}}-1\right)=E\left(e^{Z_{S t}^{(1)}}\right)-1=e^{\mu_{1}+(1 / 2) \sigma_{1}^{2}}-1 .
$$

We assume the dynamics of the counterparty's asset value $V_{t}$ are also driven by the following regime-switching jumpdiffusion process.

$$
\begin{aligned}
\frac{d V_{t}}{V_{t-}}= & \left(\beta_{t}-k_{2} \lambda_{V t}^{*}\right) d t+\sigma_{V t} d W_{t}^{(2)} \\
& +\left(e^{Z_{V t-}^{(2)}}-1\right) d M_{t}^{(2)},
\end{aligned}
$$

where $W_{t}^{(2)}$ is a standard Brownian motion on $(\Omega, \mathscr{F}, P)$. We assume the expected return rate $\left(\beta_{t}\right)_{t \in \mathscr{T}}$ and the volatility $\left(\sigma_{V t}\right)_{t \in \mathscr{T}}$ of the counterparty's asset value $V_{t}$ depend on $\left\{U_{t}\right\}_{t \in \mathscr{T}}$ and are defined by

$$
\begin{gathered}
\beta_{t}=\left\langle\beta, U_{t}\right\rangle, \\
\sigma_{V t}=\left\langle\sigma_{V}, U_{t}\right\rangle,
\end{gathered}
$$

where $\beta=\left(\beta_{1}, \beta_{2}, \ldots, \beta_{N}\right) \in \mathbf{R}^{N}$ and $\sigma_{V}=\left(\sigma_{V 1}, \sigma_{V 2}, \ldots\right.$, $\left.\sigma_{V N}\right) \in \mathbf{R}^{N}$ with $\sigma_{V i}>0$ for each $i=1,2, \ldots, N . M_{t}^{(2)}$ is also a Poisson process with corresponding intensity $\lambda_{V t}^{*}$. In addition, shocks to $V_{t}$ also include the individual component and the common component. Both the jump term $M_{t}^{(2)}$ and the intensity $\lambda_{V t}^{*}$ consist of two parts,

$$
\begin{gathered}
M_{t}^{(2)}=N_{t}^{(2)}+N_{t}, \\
\lambda_{V t}^{*}=\lambda_{V t}+\lambda_{t},
\end{gathered}
$$

where $N_{t}^{(2)}$ is a Poisson process with intensity $\lambda_{V t}$, which is independent of $N_{t}$ and $N_{t}^{(1)}$. The intensities $\lambda_{V t}^{*}, \lambda_{V t}$, and $\lambda_{t}$ also depend on the states of $\left\{U_{t}\right\}_{t \in \mathscr{T}}$; they are given by

$$
\begin{aligned}
& \lambda_{V t}^{*}=\left\langle\lambda_{V}^{*}, U_{t}\right\rangle, \\
& \lambda_{V t}=\left\langle\lambda_{V}, U_{t}\right\rangle,
\end{aligned}
$$

where $\lambda_{V}^{*}=\left(\lambda_{V 1}^{*}, \lambda_{V 2}^{*}, \ldots, \lambda_{V N}^{*}\right) \in \mathbf{R}^{N}$ and $\lambda_{V}=\left(\lambda_{V 1}, \lambda_{V 2}\right.$, $\left.\ldots, \lambda_{V N}\right) \in \mathbf{R}^{N}$. If the jump occurs at time $t$, the jump amplitude of $V_{t}$ is controlled by $Z_{V t}^{(2)}$. For $t \neq s$, we assume that $Z_{V t}^{(2)}$ and $Z_{V s}^{(2)}$ are independently and identically distributed with distribution

$$
Z_{V t}^{(2)} \sim N\left(\mu_{2}, \sigma_{2}^{2}\right)
$$

The mean percentage jump size is given by

$$
k_{2}=E\left(e^{Z_{V t}^{(2)}}-1\right)=E\left(e^{Z_{V t}^{(2)}}\right)-1=e^{\mu_{2}+(1 / 2) \sigma_{2}^{2}}-1
$$

$W_{t}^{(1)}$ and $W_{t}^{(2)}$ have the correlation coefficient $\rho$. Moreover, we assume that $\left(W_{t}^{(1)}, W_{t}^{(2)}\right), N_{t}, N_{t}^{(1)}, N_{t}^{(2)}, Z_{S t}^{(1)}$, and $Z_{V t}^{(2)}$ are mutually independent.

\section{Vulnerable European Option Pricing Considering the Market Prices of Common Jump Risks}

3.1. Equivalent Martingale Measure via Esscher Transform. The financial market described by a regime-switching model and the jump components is incomplete; hence the riskneutral measure is not uniquely determined. In order to obtain the valuation of vulnerable European options, we need to choose a special martingale measure. Esscher transform, which was first introduced by Gerber and Shiu [19], is a method of selecting the equivalent martingale measure and has extensive applications in the fields of finance and insurance. In this subsection, we adopt the random Esscher transform of Elliott et al. [20] to identify an equivalent martingale measure.

Here, we divide jump risks into two parts and price common jump risks caused by market factors in the model while neglecting the jump risks caused by the assets themselves.

First, we define two processes $Y_{1 t}$ and $Y_{2 t}$ as follows:

$$
\begin{aligned}
Y_{1 t}= & \ln \frac{S_{t}}{S_{0}} \\
= & \int_{0}^{t}\left(\alpha_{s}-k_{1} \lambda_{S s}^{*}-\frac{1}{2} \sigma_{S s}^{2}\right) d s+\int_{0}^{t} \sigma_{S s} d W_{s}^{(1)} \\
& +\int_{0}^{t} Z_{S s-}^{(1)} d N_{s}^{(1)}+\int_{0}^{t} Z_{S s-}^{(1)} d N_{s}, \\
Y_{2 t}= & \ln \frac{V_{t}}{V_{0}} \\
= & \int_{0}^{t}\left(\beta_{s}-k_{2} \lambda_{V s}^{*}-\frac{1}{2} \sigma_{V s}^{2}\right) d s+\int_{0}^{t} \sigma_{V s} d W_{s}^{(2)} \\
& +\int_{0}^{t} Z_{V s-}^{(2)} d N_{s}^{(2)}+\int_{0}^{t} Z_{V s-}^{(2)} d N_{s} .
\end{aligned}
$$

For simplicity, let

$$
\begin{aligned}
& Y_{1 t}=C_{1 t}+J_{1 t}+J_{t}, \\
& Y_{2 t}=C_{2 t}+J_{2 t}+J_{t}^{\prime},
\end{aligned}
$$


4

Discrete Dynamics in Nature and Society

where $C_{i t}, J_{i t}(i=1,2)$ and $J_{t}\left(J_{t}^{\prime}\right)$ are the continuous diffusive part, common jump part, and individual jump part, respectively, of $Y_{i t}, i=1,2$. They have the following forms:

$$
\begin{aligned}
C_{1 t} & =\int_{0}^{t}\left(\alpha_{s}-k_{1} \lambda_{S s}^{*}-\frac{1}{2} \sigma_{S s}^{2}\right) d s+\int_{0}^{t} \sigma_{S s} d W_{s}^{(1)}, \\
J_{t} & =\int_{0}^{t} Z_{S s^{-}}^{(1)} d N_{s}^{(1)}, \\
J_{1 t} & =\int_{v}^{t} Z_{S s-}^{(1)} d N_{s}, \\
C_{2 t} & =\int_{0}^{t}\left(\beta_{s}-k_{2} \lambda_{V s}^{*}-\frac{1}{2} \sigma_{V s}^{2}\right) d s+\int_{0}^{t} \sigma_{V s} d W_{s}^{(2)},
\end{aligned}
$$

$$
\begin{aligned}
J_{t}^{\prime} & =\int_{0}^{t} Z_{V s^{-}}^{(2)} d N_{s}^{(2)}, \\
J_{2 t} & =\int_{0}^{t} Z_{V s^{-}}^{(2)} d N_{s^{\prime} .}
\end{aligned}
$$

Let $\left\{\mathscr{F}_{t}^{U}\right\}_{t \in \mathscr{T}}$ and $\left\{\mathscr{F}_{t}^{i}\right\}_{t \in \mathscr{T}}$ denote the $P$-augmentations of natural filtration generated by $U$ and $Y_{i}(i=1,2)$, respectively. For each $i=1,2$, set $\mathscr{G}_{t}^{i}=\mathscr{F}_{t}^{U} \vee \mathscr{F}_{t}^{i}$. For any $t \in[0, T]$, let $\mathscr{H}_{t}=\mathscr{G}_{t}^{1} \vee \mathscr{G}_{t}^{2}$. Then, the random Escher transform $Q_{\theta} \sim P$ on $\mathscr{H}_{T}$ with regime-switching parameters $\theta_{1 t}=\left(\theta_{1 t}^{c_{1}}, \theta_{1 t-}^{J_{1}}\right)_{t \in[0, T]}$ and $\theta_{2 t}=\left(\theta_{2 t}^{c_{2}}, \theta_{2 t-}^{J_{2}}\right)_{t \in[0, T]}$ is given as follows:

$$
\left.\frac{d Q_{\theta}}{d P}\right|_{\mathscr{H}_{T}}=\frac{\exp \left(\int_{0}^{T} \theta_{1 t}^{\mathcal{c}_{1}} d C_{1 t}+\int_{0}^{T} \theta_{1 t-}^{J_{1}} d J_{1 t}+\int_{0}^{T} \theta_{2 t}^{c_{2}} d C_{2 t}+\int_{0}^{T} \theta_{2 t-}^{J_{2}} d J_{2 t}\right)}{E\left[\exp \left(\int_{0}^{T} \theta_{1 t}^{c_{1}} d C_{1 t}+\int_{0}^{T} \theta_{1 t-}^{J_{1}} d J_{1 t}+\int_{0}^{T} \theta_{2 t}^{c_{2}} d C_{2 t}+\int_{0}^{T} \theta_{2 t-}^{J_{2}} d J_{2 t}\right) \mid \mathscr{F}_{T}^{U}\right]},
$$

where $E[\cdot]$ denote the expectation under measure $P$. For $m \in$ $\left\{c_{1}, J_{1}\right\}, \theta_{1 t}^{m}=\left\langle\theta_{1}^{m}, U_{t}\right\rangle$, and $\theta_{1}^{m}=\left(\theta_{11}^{m}, \theta_{12}^{m}, \ldots, \theta_{1 N}^{m}\right) \in \mathbf{R}^{N}$, and for $m \in\left\{c_{2}, J_{2}\right\}, \theta_{2 t}^{m}=\left\langle\theta_{2}^{m}, U_{t}\right\rangle$ and $\theta_{2}^{m}=\left(\theta_{21}^{m}, \theta_{22}^{m}, \ldots, \theta_{2 N}^{m}\right) \in$ $\mathbf{R}^{N}$. Since

$$
\begin{aligned}
E & {\left[\operatorname { e x p } \left(\int_{0}^{T} \theta_{1 t}^{c_{1}} d C_{1 t} \int_{0}^{T} \theta_{1 t-}^{J_{1}} d J_{1 t}+\int_{0}^{T} \theta_{2 t}^{c_{2}} d C_{2 t}\right.\right.} \\
& \left.\left.+\int_{0}^{T} \theta_{2 t-}^{J_{2}} d J_{2 t}\right) \mid \mathscr{F}_{T}^{U}\right] \\
& =\exp \left(\int_{0}^{T} \theta_{1 t}^{c_{1}}\left(\alpha_{t}-k_{1} \lambda_{S t}^{*}-\frac{1}{2} \sigma_{S t}^{2}\right) d t\right. \\
& +\int_{0}^{T} \theta_{2 t}^{c_{2}}\left(\beta_{t}-k_{2} \lambda_{V t}^{*}-\frac{1}{2} \sigma_{V t}^{2}\right) d t+\frac{1}{2} \\
& \cdot \int_{0}^{T}\left(\left(\theta_{1 t}^{c_{1}}\right)^{2} \sigma_{S t}^{2}+\left(\theta_{2 t}^{c_{2}}\right)^{2} \sigma_{V t}^{2}+2 \rho \theta_{1 t}^{c_{1}} \sigma_{S t} \theta_{2 t}^{c_{2}} \sigma_{V t}\right) d t \\
& \left.+\int_{0}^{T} \lambda_{t}\left(e^{\left(\theta_{1 t-}^{I_{1}} \mu_{1}+\theta_{2 t-}^{J_{2}} \mu_{2}\right)+(1 / 2)\left(\left(\theta_{1 t-}^{I_{1}} \sigma_{1}\right)^{2}+\left(\theta_{2 t-}^{J_{2}} \sigma_{2}\right)^{2}\right)}-1\right) d t\right)
\end{aligned}
$$

then

$$
\begin{aligned}
& \left.\frac{d Q_{\theta}}{d P}\right|_{\mathscr{H}_{T}}=\exp \left(\int_{0}^{T} \theta_{1 t}^{c_{1}} \sigma_{S t} d W_{t}^{(1)}+\int_{0}^{T} \theta_{2 t}^{c_{2}} \sigma_{V t} d W_{t}^{(2)}-\frac{1}{2}\right. \\
& \left.\cdot \int_{0}^{T}\left(\left(\theta_{1 t}^{c_{1}}\right)^{2} \sigma_{S t}^{2}+\left(\theta_{2 t}^{c_{2}}\right)^{2} \sigma_{V t}^{2}+2 \rho \theta_{1 t}^{c_{1}} \sigma_{S t} \theta_{2 t}^{c_{2}} \sigma_{V t}\right) d t\right) \\
& \times \exp \left(\int_{0}^{T}\left(\theta_{1 t-}^{J_{1}} Z_{S t-}^{(1)}+\theta_{2 t-}^{J_{2}} Z_{V t-}^{(2)}\right) d N_{t}\right. \\
& \left.-\int_{0}^{T} \lambda_{t}\left(e^{\left(\theta_{1 t-}^{I_{1}} \mu_{1}+\theta_{2 t-}^{J_{2}} \mu_{2}\right)+(1 / 2)\left(\left(\theta_{1 t-}^{J_{1}} \sigma_{1}\right)^{2}+\left(\theta_{2 t-}^{J_{2}} \sigma_{2}\right)^{2}\right)}-1\right) d t\right) .
\end{aligned}
$$

By the fundamental theorem of asset pricing, the absence of arbitrage opportunities is "essentially" equivalent to the existence of an equivalent martingale measure under which the discounted stock price process is a martingale. Following Elliott et al. [20], due to the presence of the uncertainty generated by the Markov chain process, the martingale condition is obtained by considering an enlarged filtration $\mathscr{H}$. The following theorem presents the result.

Theorem 1. The martingale conditions are satisfied if and only if

$$
\begin{aligned}
& \alpha_{t}-r_{t}-k_{1} \lambda_{t}+\theta_{1 t}^{c_{1}} \sigma_{S t}^{2}+\rho \sigma_{S t} \theta_{2 t}^{c_{2}} \sigma_{V t}+\tilde{\lambda}_{t} k_{S t}^{\theta_{1 t-}^{I_{1}}}=0, \\
& \beta_{t}-r_{t}-k_{2} \lambda_{t}+\theta_{2 t}^{c_{2}} \sigma_{V t}^{2}+\rho \sigma_{S t} \theta_{1 t}^{c_{1}} \sigma_{V t}+\tilde{\lambda}_{t} k_{V t}^{\theta_{2 t-}^{I_{2}}}=0,
\end{aligned}
$$

where

$$
\begin{aligned}
\tilde{\lambda}_{t} & =\lambda_{t} e^{\left(\theta_{1 t-}^{I_{1}} \mu_{1}+\theta_{2 t-}^{I_{2}} \mu_{2}\right)+(1 / 2)\left(\left(\theta_{1 t-}^{I_{1}} \sigma_{1}\right)^{2}+\left(\theta_{2 t-}^{I_{2}} \sigma_{2}\right)^{2}\right)}, \\
k_{S t}^{\theta_{1 t-}^{I_{1}}} & =\left(k_{1}+1\right) e^{\theta_{1 t-}^{J_{1}} \sigma_{1}^{2}}-1, \\
k_{V t}^{\theta_{2 t-}^{I_{2}}} & =\left(k_{2}+1\right) e^{\theta_{2 t-}^{J_{2}} \sigma_{2}^{2}}-1 .
\end{aligned}
$$

Proof. Let

$$
\begin{aligned}
& M_{S t}^{C}=\int_{u}^{t}\left(1+\theta_{1 s}^{c_{1}}\right) \sigma_{S s} d W_{s}^{(1)}+\int_{u}^{t} \theta_{2 s}^{c_{2}} \sigma_{V s} d W_{s}^{(2)}-\frac{1}{2} \\
& \cdot \int_{u}^{t}\left(\left(1+\theta_{1 s}^{c_{1}}\right)^{2} \sigma_{S s}^{2}+\left(\theta_{2 s}^{c_{2}}\right)^{2} \sigma_{V s}^{2}\right. \\
& \left.\quad+2 \rho\left(1+\theta_{1 s}^{c_{1}}\right) \sigma_{S s} \theta_{2 s}^{c_{2}} \sigma_{V s}\right) d s, \\
& M_{S t}^{J_{1}}=\int_{\mathcal{u}}^{t}\left(\left(1+\theta_{1 s^{-}}^{J_{1}}\right) Z_{S s^{-}}^{(1)}+\theta_{2 s_{-}}^{J_{2}} Z_{V s-}^{(2)}\right) d N_{s} \\
& \quad-\int_{u}^{t} \lambda_{s}\left(e^{\left(\left(1+\theta_{1 s-}^{J_{1}}\right) \mu_{1}+\theta_{2 s-}^{J_{2}} \mu_{2}\right)+(1 / 2)\left(\left(1+\theta_{1 s^{-}}^{J_{1}}\right)^{2} \sigma_{1}^{2}+\left(\theta_{2 s_{-}-}^{J_{2}} \sigma_{2}\right)^{2}\right)}\right. \\
& \quad-1) d s .
\end{aligned}
$$


Then, by Bayes' rule, we have

$$
\begin{aligned}
& E^{\mathrm{Q}_{\theta}}\left[e^{-\int_{0}^{t} r_{s} d s} S_{t} \mid \mathscr{H}_{u}\right]=\frac{E\left[e^{-\int_{0}^{t} r_{s} d s} S_{t} \times \frac{d Q_{\theta}}{d P} \mid \mathscr{H}_{u}\right]}{E\left[\frac{d Q_{\theta}}{d P} \mid \mathscr{H}_{u}\right]}=e^{-\int_{0}^{u} r_{s} d s} S_{u} E\left\{\operatorname { e x p } \left(\int_{u}^{t}\left(\alpha_{s}-r_{s}-k_{1} \lambda_{S s}^{*}+\theta_{1 s}^{c_{1}} \sigma_{S s}^{2}+\rho \sigma_{S s} \theta_{2 s}^{c_{2}} \sigma_{V s}\right) d s\right.\right. \\
& \left.+M_{S t}^{c}\right) \times \exp \left[-\int_{\mathcal{U}}^{t} \lambda_{s}\left(e^{\left(\theta_{1 s-}^{I_{1}} \mu_{1}+\theta_{2 s-}^{I_{2}} \mu_{2}\right)+(1 / 2)\left(\left(\theta_{1 s-}^{I_{1}} \sigma_{1}\right)^{2}+\left(\theta_{2 s-}^{I_{2}} \sigma_{2}\right)^{2}\right)}-1\right) d s\right. \\
& \left.\left.+\int_{u}^{t} \lambda_{s}\left(e^{\left(\left(1+\theta_{1 s}^{I_{1}}\right) \mu_{1}+\theta_{2 s}^{I_{2}} \mu_{2}\right)+(1 / 2)\left(\left(1+\theta_{1 s-}^{I_{1}}\right)^{2} \sigma_{1}^{2}+\left(\theta_{2 s-}^{I_{2}} \sigma_{2}\right)^{2}\right)}-1\right) d s+M_{S t}^{J_{1}}\right] \times \exp \left(\int_{u}^{t} Z_{S s-}^{(1)} d N_{s}^{(1)}\right)\right\}=e^{-\int_{0}^{u} r_{s} d s} S_{u} \\
& \cdot \exp \left(\int_{\mathcal{U}}^{t}\left(\alpha_{s}-r_{s}-k_{1} \lambda_{S s}^{*}+\theta_{1 s}^{c_{1}} \sigma_{S s}^{2}+\rho \sigma_{S s} \theta_{2 s}^{c_{2}} \sigma_{V s}\right) d s\right) \times \exp \left[-\int_{\mathcal{U}}^{t} \lambda_{s}\left(e^{\left(\theta_{1 s}^{I_{1}}-\mu_{1}+\theta_{2 s-}^{J_{2}} \mu_{2}\right)+(1 / 2)\left(\left(\theta_{1 s-}^{I_{1}} \sigma_{1}\right)^{2}+\left(\theta_{2 s-}^{I_{2}} \sigma_{2}\right)^{2}\right)}-1\right) d s\right. \\
& \left.+\int_{u}^{t} \lambda_{s}\left(e^{\left(\left(1+\theta_{1 s-}^{I_{1}}\right) \mu_{1}+\theta_{2 s-}^{I_{2}} \mu_{2}\right)+(1 / 2)\left(\left(1+\theta_{1 s-}^{I_{1}}\right)^{2} \sigma_{1}^{2}+\left(\theta_{2 s-}^{I_{2}} \sigma_{2}\right)^{2}\right)}-1\right) d s\right] \times \exp \left(\int_{\mathcal{u}}^{t} \lambda_{S s}\left(e^{\mu_{1}+(1 / 2) \sigma_{1}^{2}}-1\right) d s\right) .
\end{aligned}
$$

The martingale condition

$$
E^{\mathrm{Q}_{\theta}}\left[e^{-\int_{0}^{t} r_{s} d s} S_{t} \mid \mathscr{H}_{u}\right]=e^{-\int_{0}^{u} r_{s} d s} S_{u}
$$

is satisfied if and only if

$$
\alpha_{t}-r_{t}-k_{1} \lambda_{t}+\theta_{1 t}^{c_{1}} \sigma_{S t}^{2}+\rho \sigma_{S t} \theta_{2 t}^{c_{2}} \sigma_{V t}+\tilde{\lambda}_{t} k_{S t}^{\theta_{1 t-}^{I_{1}}}=0
$$

where

$$
\begin{aligned}
\tilde{\lambda}_{t} & =\lambda_{t} e^{\left(\theta_{1 t}^{1} \mu_{1}+\theta_{2 t}^{J_{2}} \mu_{2}\right)+(1 / 2)\left(\left(\theta_{1 t-}^{J_{1}} \sigma_{1}\right)^{2}+\left(\theta_{2 t}^{J_{2}} \sigma_{2}\right)^{2}\right)}, \\
k_{S t}^{\theta_{1 t}^{J_{1}}} & =\left(k_{1}+1\right) e^{\theta_{1 t-}^{J_{1}} \sigma_{1}^{2}}-1 .
\end{aligned}
$$

Similarly, since

$$
E^{\mathrm{Q}_{\theta}}\left[e^{-\int_{0}^{t} r_{s} d s} V_{t} \mid \mathscr{H}_{u}\right]=e^{-\int_{0}^{u} r_{s} d s} V_{u},
$$

it follows that

$$
\beta_{t}-r_{t}-k_{2} \lambda_{t}+\theta_{2 t}^{c_{2}} \sigma_{V t}^{2}+\rho \sigma_{S t} \theta_{1 t}^{c_{1}} \sigma_{V t}+\tilde{\lambda}_{t} k_{V t}^{\theta_{2 t-}^{J_{2}}}=0
$$

where $\tilde{\lambda}_{t}=\lambda_{t} e^{\left(\theta_{1 t-}^{J_{1}} \mu_{1}+\theta_{2 t}^{J_{2}} \mu_{2}\right)+(1 / 2)\left(\left(\theta_{1 t-}^{I_{1}} \sigma_{1}\right)^{2}+\left(\theta_{2 t}^{J_{2}} \sigma_{2}\right)^{2}\right)}$ and $k_{V t}^{\theta_{2 t}^{J_{2}}}=$ $\left(k_{2}+1\right) e^{\theta_{2 t}^{J_{2}} \sigma_{2}^{2}}-1$.

Proposition 2. Conditional on $\mathscr{F}_{T}^{U}$ and under the equivalent martingale measure $Q_{\theta}, S_{t}$ and $V_{t}$ follow stochastic differential equations:

$$
\frac{d S_{t}}{S_{t-}}=\left(r_{t}-k_{1} \lambda_{S t}-\widetilde{\lambda}_{t} k_{S t}^{\theta_{1 t}^{J_{1}}}\right) d t+\sigma_{S t} d \widetilde{W}_{t}^{(1)}
$$

$$
\begin{aligned}
& +\left(e^{Z_{s t-}^{(1)}}-1\right) d N_{t}^{(1)}+\left(e^{\widetilde{Z}_{s t-}^{(1)}}-1\right) d \widetilde{N}_{t}, \\
\frac{d V_{t}}{V_{t-}}= & \left(r_{t}-k_{2} \lambda_{V t}-\widetilde{\lambda}_{t} k_{V t}^{\theta_{2 t}^{J_{2}}}\right) d t+\sigma_{V t} d \widetilde{W}_{t}^{(2)} \\
& +\left(e^{Z_{V t-}^{(2)}}-1\right) d N_{t}^{(2)}+\left(e^{\widetilde{Z}_{V t-}^{(2)}}-1\right) d \widetilde{N}_{t},
\end{aligned}
$$

where $\theta_{1 t}^{c_{1}}, \theta_{1 t-}^{J_{1}}, \theta_{2 t}^{c_{2}}$, and $\theta_{2 t-}^{J_{2}}$ are given by (21). Moreover,

$$
\begin{aligned}
& \widetilde{W}_{t}^{(1)}=W_{t}^{(1)}-\int_{0}^{t} \sigma_{S s} \theta_{1 s}^{c_{1}} d s-\rho \int_{0}^{t} \sigma_{V s} \theta_{2 s}^{c_{2}} d s, \\
& \widetilde{W}_{t}^{(2)}=W_{t}^{(2)}-\int_{0}^{t} \sigma_{V s} \theta_{2 s}^{c_{2}} d s-\rho \int_{0}^{t} \sigma_{S s} \theta_{1 s}^{c_{1}} d s
\end{aligned}
$$

are two standard $Q_{\theta}$ Brownian motions. $\widetilde{N}_{t}$ is still a Poisson process under the measure $Q_{\theta}$ with the intensity rate $\widetilde{\lambda}_{t} . \widetilde{\lambda}_{t}$ is given as follows:

$$
\tilde{\lambda}_{t}=\lambda_{t} e^{\left(\theta_{1 t-}^{I_{1}} \mu_{1}+\theta_{2 t}^{I_{2}} \mu_{2}\right)+(1 / 2)\left(\left(\theta_{1 t-}^{I_{1}} \sigma_{1}\right)^{2}+\left(\theta_{2 t-}^{I_{2}} \sigma_{2}\right)^{2}\right)},
$$

and the mean percentage jump sizes of $\widetilde{Z}_{S t-}^{(1)}$ and $\widetilde{Z}_{V t-}^{(2)}$ are

$$
\begin{aligned}
& k_{S t}^{\theta_{1 t-}^{J_{1}}}=\left(k_{1}+1\right) e^{\theta_{1 t-}^{J_{1}} \sigma_{1}^{2}}-1, \\
& k_{V t}^{\theta_{2 t}^{I_{2}}}=\left(k_{2}+1\right) e^{\theta_{2 t-}^{I_{2}} \sigma_{2}^{2}}-1 .
\end{aligned}
$$

Proof. Under the conditions of $\mathscr{F}_{T}^{U}$, we use Girsanov's theorem and (20) to obtain $\widetilde{W}_{t}^{(1)}, \widetilde{W}_{t}^{(2)}$ which are two standard $Q_{\theta}$ Brownian motions with the correlation coefficient $\rho$. Note that the parameters describing the common jumps in the two risky assets values have been changed. Now, we prove the formulae of (32) and (33). 


$$
\begin{aligned}
& E^{Q_{\theta}}\left[\exp \left(i u \int_{0}^{T} Z_{S t-}^{(1)} d N_{t}\right) \mid \mathscr{F}_{T}^{U}\right]=E\left[\exp \left(i u \int_{0}^{T} Z_{S t-}^{(1)} d N_{t}\right) \times \frac{d Q_{\theta}}{d P} \mid \mathscr{F}_{T}^{U}\right]
\end{aligned}
$$

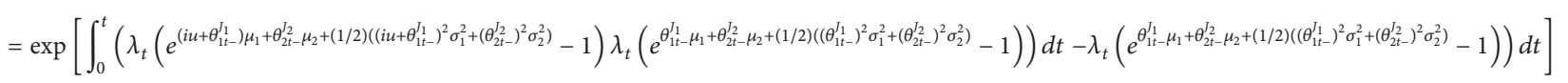

$$
\begin{aligned}
& =\exp \left(\int_{0}^{T} \tilde{\lambda}_{t} \int_{-\infty}^{+\infty}\left(e^{i u z}-1\right) \tilde{f}_{U_{S}}(z) d t\right) \text {. }
\end{aligned}
$$

Hence, under the condition $\mathscr{F}_{T}^{U}$ and the probability measure $Q_{\theta}$, the intensity rate of $N_{t}$ is $\tilde{\lambda}_{t}=$ $\lambda_{t} e^{\left(\theta_{1 t-}^{J_{1}} \mu_{1}+\theta_{2 t-}^{I_{2}} \mu_{2}\right)+(1 / 2)\left(\left(\theta_{1 t-}^{I_{1}} \sigma_{1}\right)^{2}+\left(\theta_{2 t-}^{I_{2}} \sigma_{2}\right)^{2}\right)}$. The density function of $Z_{S t-}^{(1)}$ is

$$
\tilde{f}_{U_{S}}(z)=\frac{1}{\sqrt{2 \pi} \sigma_{1}} e^{-\left(z-\left(\mu_{1}+\theta_{1 t-}^{J_{1}} \sigma_{1}^{2}\right)\right)^{2} / 2 \sigma_{1}^{2}}, \quad z \in(-\infty, \infty) .
$$

Similarly, we can also prove that the density function of $Z_{V t-}^{(2)}$ is

$$
\begin{aligned}
& \tilde{f}_{U_{V}}(z)=\frac{1}{\sqrt{2 \pi} \sigma_{2}} e^{-\left(z-\left(\mu_{2}+\theta_{2 t-}^{I_{2}} \sigma_{2}^{2}\right)\right)^{2} / 2 \sigma_{2}^{2},} \\
& \\
& z \in(-\infty, \infty) .
\end{aligned}
$$

Note that the risk-neutral probability measure $Q_{\theta}$ is selected by the Esscher transform, and the probability law of the Markov chain $U$ remains the same after the measure change; that is, under $Q_{\theta}, U$ still has the same semimartingale dynamics. For each $0 \leq t \leq T$, we find that the solutions of (21) are not unique. Hence, a special case needs to be considered to obtain an equivalent martingale measure. We suppose

$$
\begin{aligned}
\alpha_{t}-r_{t}-k_{1} \lambda_{t}+\theta_{1 t}^{c_{1}} \sigma_{S t}^{2}+\rho \sigma_{S t} \theta_{2 t}^{c_{2}} \sigma_{V t} & =0, \\
\tilde{\lambda}_{t}\left(e^{\mu_{1}+(1 / 2) \sigma_{1}^{2}+\theta_{1 t-}^{J_{1}} \sigma_{1}^{2}}-1\right) & =0, \\
\beta_{t}-r_{t}-k_{2} \lambda_{t}+\theta_{2 t}^{c_{2}} \sigma_{V t}^{2}+\rho \sigma_{S t} \theta_{1 t}^{c_{1}} \sigma_{V t} & =0, \\
\tilde{\lambda}_{t}\left(e^{\mu_{2}+(1 / 2) \sigma_{2}^{2}+\theta_{2 t-}^{I_{2}} \sigma_{2}^{2}}-1\right) & =0 .
\end{aligned}
$$

Then the following corollary gives a pair of solutions for the martingale condition.

Corollary 3. The martingale conditions (21) are satisfied if the parameters are given as follows:

$$
\begin{aligned}
& \theta_{1 t}^{c_{1} *}=\frac{\left(\beta_{t}-r_{t}-k_{2} \lambda_{t}\right) \rho \sigma_{S t}-\left(\alpha_{t}-r_{t}-k_{1} \lambda_{t}\right) \sigma_{V t}}{\left(1-\rho^{2}\right) \sigma_{S t}^{2} \sigma_{V t}}, \\
& \theta_{2 t}^{c_{2} *}=\frac{\left(\alpha_{t}-r_{t}-k_{1} \lambda_{t}\right) \rho \sigma_{V t}-\left(\beta_{t}-r_{t}-k_{2} \lambda_{t}\right) \sigma_{S t}}{\left(1-\rho^{2}\right) \sigma_{V t}^{2} \sigma_{S t}}, \\
& \theta_{1 t-}^{J_{1} *}=-\frac{\mu_{1}+(1 / 2) \sigma_{1}^{2}}{\sigma_{1}^{2}} \\
& \theta_{2 t-}^{J_{2}}{ }^{*}=-\frac{\mu_{2}+(1 / 2) \sigma_{2}^{2}}{\sigma_{2}^{2}}
\end{aligned}
$$

$$
\begin{aligned}
\tilde{k}_{S t}^{\theta_{1 t-}^{J_{1}}} & =\left(k_{1}+1\right) e^{\theta_{1 t-}^{J_{1}} \sigma_{1}^{2}}-1=0, \\
\tilde{k}_{V t}^{\theta_{2 t}^{J_{2}}} & =\left(k_{2}+1\right) e^{\theta_{2 t-}^{J_{2}} \sigma_{2}^{2}}-1=0, \\
\tilde{\lambda}_{t}^{*} & =\lambda_{t} \exp \left(-\frac{\mu_{1}^{2}}{2 \sigma_{1}^{2}}+\frac{\sigma_{1}^{2}}{8}\right) \times \exp \left(-\frac{\mu_{2}^{2}}{2 \sigma_{2}^{2}}+\frac{\sigma_{2}^{2}}{8}\right) .
\end{aligned}
$$

The explicit Esscher parameters in Corollary 3 will be used to the Monte Carlo simulations in Section 5.

3.2. Vulnerable European Option Pricing. As in Klein [2], we assume that default will only occur if the counterparty's asset value at maturity $V_{T}$ is less than some amount $D^{*}$. Additionally, this amount is not set to the value of the option but corresponds to the amount of the counterparty's claim $D$ at exercise time $T$. Once a credit loss occurs at exercise time $T$, only the proportion $(1-\alpha) V_{T} / D$ of the value of the option at $T$ is paid out by the counterparty. Then, the payoff of a vulnerable European call option is given by

$$
\left(S_{T}-K\right)^{+} \mathbf{1}_{\left\{V_{T} \geq D^{*}\right\}}+\frac{(1-\alpha) V_{T}\left(S_{T}-K\right)^{+}}{D} \mathbf{1}_{\left\{V_{T}<D^{*}\right\}},
$$

where $K$ is the strike price of the option and $\alpha$ is the deadweight cost associated with bankruptcy, expressed as a percentage of the value of the counterparty's assets. By the risk-neutral arguments, under the risk-neutral measure $Q_{\theta}$, the price of a vulnerable European call option at time 0 is given as

$$
\begin{gathered}
C(S, V, U)=E^{Q_{\theta}}\left[e^{-\int_{0}^{T} r_{s} d s}\left(S_{T}-K\right)^{+}\right. \\
\left.\cdot\left(\mathbf{1}_{\left\{V_{T} \geq D^{*}\right\}}+\frac{(1-\alpha) V_{T}}{D} \mathbf{1}_{\left\{V_{T}<D^{*}\right\}}\right)\right] .
\end{gathered}
$$

$r_{t}$ is the risk-free rate. $C(S, V, U)$ depends on $U$ for the Markov regime-switching in our pricing model.

Under the conditions $\mathscr{G}_{T}^{\left(n_{1}, n_{2}, n_{3}\right)}:=\left\{\widetilde{N}_{T}=n, N_{T}^{(1)}=\right.$ $\left.n_{1}, N_{T}^{(2)}=n_{2}\right\}$ and $\mathscr{F}_{T}^{U}$, for $m_{1}=n+n_{1}, m_{2}=n+n_{2}$, the value of a vulnerable European call option at time 0 is given as

$$
\begin{aligned}
& C_{m_{1}, m_{2}}=E^{\mathrm{Q}_{\theta}}\left[e^{-\int_{0}^{T} r_{s} d s}\left(S_{T, m_{1}}-K\right)^{+}\right. \\
& \left.\cdot\left(\mathbf{1}_{\left\{V_{T, m_{2}} \geq D^{*}\right\}}+\frac{(1-\alpha) V_{T, m_{2}}}{D} \mathbf{1}_{\left\{V_{T, m_{2}}<D^{*}\right\}}\right)\right] .
\end{aligned}
$$


Clearly, $C_{m_{1}, m_{2}}$ represents the value of the vulnerable option given $\mathscr{F}_{T}^{U}$, conditional on $m_{1}$ and $m_{2}$ jumps on the underlying asset and the assets of the counterparty, respectively.

Therefore, the conditional vulnerable European call option price given $\mathscr{F}_{T}^{U}$ is

$$
\begin{aligned}
C^{*} & =E^{\mathrm{Q}_{\theta}}\left[e ^ { - \int _ { 0 } ^ { T } r _ { s } d s } ( S _ { T } - K ) ^ { + } \left(\mathbf{1}_{\left\{V_{T} \geq D^{*}\right\}}+\frac{(1-\alpha) V_{T}}{D}\right.\right. \\
& \left.\left.\cdot \mathbf{1}_{\left\{V_{T}<D^{*}\right\}}\right) \mid \mathscr{F}_{T}^{U}\right] \\
= & \sum_{n=0}^{\infty} \sum_{n_{1}=0}^{\infty} \sum_{n_{2}=0}^{\infty} \mathrm{Q}\left(\widetilde{N}_{T}=n, N_{T}^{(1)}=n_{1}, N_{T}^{(2)}=n_{2}\right) \\
& \cdot C_{m_{1}, m_{2}} .
\end{aligned}
$$

Let $J_{i}$ denote the occupation time of $U:=\left\{U_{t}\right\}_{t \in \mathscr{T}}$ in state $i$ over the time duration $[0, T]$, where $t \in \mathscr{T}$. Then

$$
\begin{aligned}
& \bar{R}_{T}=\frac{1}{T} \int_{0}^{T} r_{t} d t=\frac{1}{T} \sum_{i=1}^{N} r_{i} J_{i}, \\
& \bar{U}_{1 T}=\frac{1}{T} \int_{0}^{T} \sigma_{S t}^{2} d t=\frac{1}{T} \sum_{i=1}^{N} \sigma_{S i}^{2} J_{i}, \\
& \bar{U}_{2 T}=\frac{1}{T} \int_{0}^{T} \sigma_{V t}^{2} d t=\frac{1}{T} \sum_{i=1}^{N} \sigma_{V i}^{2} J_{i}, \\
& \bar{U}_{3 T}=\frac{1}{T} \int_{0}^{T} \sigma_{S t} \sigma_{V t} d t=\frac{1}{T} \sum_{i=1}^{N} \sigma_{S i} \sigma_{V i} J_{i}, \\
& \bar{\lambda}_{S T}=\frac{1}{T} \int_{0}^{T} \lambda_{S t} d t=\frac{1}{T} \sum_{i=1}^{N} \lambda_{S i} J_{i}, \\
& \bar{\lambda}_{V T}=\frac{1}{T} \int_{0}^{T} \lambda_{V t} d t=\frac{1}{T} \sum_{i=1}^{N} \lambda_{V i} J_{i}, \\
& \overline{\tilde{\lambda}}_{T}=\frac{1}{T} \int_{0}^{T} \tilde{\lambda}_{t} d t=\frac{1}{T} \sum_{i=1}^{N} \tilde{\lambda}_{i} J_{i} .
\end{aligned}
$$

From Buffington and Elliott [21], the price of a vulnerable European call option is given by

$C(S, V, U)$

$$
=\int_{[0, T]^{N}} C^{*} \times \psi\left(J_{1}, J_{2}, \ldots, J_{N}\right) d J_{1} d J_{2} \cdots d J_{N},
$$

where $\psi\left(J_{1}, J_{2}, \ldots, J_{N}\right)$ is the joint distribution of the occupation time $\left(J_{1}, J_{2}, \ldots, J_{N}\right)$ under the condition $U_{0}=U$ and the probability measure $Q_{\theta}$. Write $J:=\left(J_{1}, J_{2}, \ldots, J_{N}\right)$ for the vector of occupation times. Let $G$ denote a diagonal matrix with the elements in the vector $\zeta=\left(\zeta_{1}, \zeta_{2}, \ldots, \zeta_{N}\right)$ as its diagonal. Then, for any $\zeta \in \mathbf{R}^{N}$, the characteristic function of $J$ under the condition $U_{0}=U$ and the martingale measure $Q_{\theta}$ is given by

$$
E_{\mathrm{Q}_{\theta}}\left[e^{i\langle\zeta, J\rangle} \mid U_{0}=U\right]=\left\langle U e^{(A+i G) T}, \mathbf{I}\right\rangle,
$$

where $i=\sqrt{-1}$ and $\mathbf{I}=(1,1, \ldots, 1) \in \mathbf{R}^{N}$. Note that $\psi\left(J_{1}\right.$, $J_{2}, \ldots, J_{N}$ ) can be completely determined by the characteristic function. As for the proof, refer to Buffington and Elliott [21].

At this point, the key problem that needs to be solved is to determine $C_{m_{1}, m_{2}}$. According to the Itô formula, the following equalities hold:

$$
\begin{aligned}
\ln S_{T}= & \ln S_{0}+\int_{0}^{T}\left(r_{t}-k_{1} \lambda_{S t}-\widetilde{\lambda}_{t} k_{S t}^{\theta_{1 t}^{J_{1}}}-\frac{1}{2} \sigma_{S t}^{2}\right) d t \\
& +\int_{0}^{T} \sigma_{S t} d \widetilde{W}_{t}^{(1)}+\sum_{i=1}^{N_{T}^{(1)}} Z_{S i}^{(1)}+\sum_{i=1}^{\widetilde{N}_{T}} \widetilde{Z}_{S i}^{(1)}, \\
\ln V_{T}= & \ln V_{0}+\int_{0}^{T}\left(r_{t}-k_{2} \lambda_{V t}-\widetilde{\lambda}_{t} k_{V t}^{\theta_{2 t}^{J_{2}}}-\frac{1}{2} \sigma_{V t}^{2}\right) d t \\
& +\int_{0}^{T} \sigma_{V t} d \widetilde{W}_{t}^{(2)}+\sum_{i=1}^{N_{T}^{(2)}} Z_{V i}^{(2)}+\sum_{i=1}^{\widetilde{N}_{T}} \widetilde{Z}_{V i}^{(2)},
\end{aligned}
$$

where $\operatorname{cov}\left(d \widetilde{W}_{t}^{(1)}, d \widetilde{W}_{t}^{(2)}\right)=\rho d t,\left(\ln S_{T, m_{1}} / \ln S_{0}, \ln V_{T, m_{2}} /\right.$ $\ln V_{0}$ ) is bivariate normally distributed under the conditions $\mathbb{G}_{T}^{\left(n, n_{1}, n_{2}\right)}:=\left\{\widetilde{N}_{T}=n, N_{T}^{(1)}=n_{1}, N_{T}^{(2)}=n_{2}\right\}$ and $\mathscr{F}_{T}^{U}$ with the following properties:

$$
\begin{aligned}
& M_{S}\left(m_{1}\right)=E\left(\frac{\ln S_{T, m_{1}}}{\ln S_{0}}\right) \\
& =\int_{0}^{T}\left(r_{t}-k_{1} \lambda_{S t}-\widetilde{\lambda}_{t} k_{S t}^{\theta_{1 t-}^{I_{1}}}-\frac{1}{2} \sigma_{S t}^{2}\right) d t+m_{1} \mu_{1} \\
& +\sigma_{1}^{2} \sum_{i=1}^{n} \theta_{1 t_{i}-}^{I_{1}}, \\
& M_{V}\left(m_{2}\right)=E\left(\frac{\ln V_{T, m_{2}}}{\ln V_{0}}\right) \\
& =\int_{0}^{T}\left(r_{t}-k_{2} \lambda_{V t}-\tilde{\lambda}_{t} k_{V t}^{\theta_{2 t-}^{I_{2}}}-\frac{1}{2} \sigma_{V t}^{2}\right) d t+m_{2} \mu_{2} \\
& \quad+\sigma_{2}^{2} \sum_{i=1}^{n} \theta_{2 t_{i}-}^{I_{2}}, \\
& \operatorname{cov}\left(\frac{\ln S_{T, m_{1}}}{\ln S_{0}}, \frac{\ln V_{T, m_{2}}}{\ln V_{0}}\right)=\int_{0}^{T} \rho \sigma_{S t} \sigma_{V t} d t .
\end{aligned}
$$

Let

$$
\begin{aligned}
& \frac{\ln S_{T, m_{1}}}{\ln S_{0}}=M_{S}\left(m_{1}\right)+\sqrt{\int_{0}^{T} \sigma_{S t}^{2} d t+m_{1} \sigma_{1}^{2}} \xi_{1}, \\
& \frac{\ln V_{T, m_{2}}}{\ln V_{0}}=M_{V}\left(m_{2}\right)+\sqrt{\int_{0}^{T} \sigma_{V t}^{2} d t+m_{2} \sigma_{2}^{2}} \xi_{2},
\end{aligned}
$$

where $\xi_{1}$ and $\xi_{2}$ are standard normal random variables and the correlation coefficient is

$$
\bar{\rho}\left(m_{1}, m_{2}\right)=\frac{\bar{U}_{3 T} T}{\sqrt{\bar{U}_{1 T} T+m_{1} \sigma_{1}^{2}} \sqrt{\bar{U}_{2 T} T+m_{2} \sigma_{2}^{2}}} \rho .
$$


Now we need to calculate $C_{m_{1}, m_{2}}$, and the derivation process can be referred to Tian et al. [12].

$$
\begin{aligned}
& C_{m_{1}, m_{2}}=e^{-\bar{R}_{T} T} E^{\mathrm{Q}_{\theta}}\left[\left(S_{T, m_{1}}-K\right)^{+}\right. \\
& \left.\cdot\left(\mathbf{1}_{\left\{V_{T, m_{2}} \geq D *\right\}}+\frac{1-\alpha}{D} V_{T, m_{2}} \mathbf{1}_{\left\{V_{T, m_{2}}<D^{*}\right\}}\right)\right] \\
& =e^{-\bar{R}_{T} T}\left[A_{1}\left(m_{1}, m_{2}\right)+A_{2}\left(m_{1}, m_{2}\right)\right. \\
& \left.+A_{3}\left(m_{1}, m_{2}\right)+A_{4}\left(m_{1}, m_{2}\right)\right],
\end{aligned}
$$

where

$$
\begin{aligned}
& A_{1}\left(m_{1}, m_{2}\right)=E^{\mathrm{Q}_{\theta}}\left[S_{T, m_{1}} \mathbf{1}_{\left\{S_{T}, m_{1} \geq K, V_{T, m_{2} \geq D *}\right\}}\right], \\
& A_{2}\left(m_{1}, m_{2}\right)=-K E^{\mathrm{Q}_{\theta}}\left[\mathbf{1}_{\left\{S_{T}, m_{1} \geq K, V_{T, m_{2} \geq D *}\right\}}\right], \\
& A_{3}\left(m_{1}, m_{2}\right) \\
& \quad=\frac{1-\alpha}{D} E^{\mathrm{Q}_{\theta}}\left[S_{T, m_{1}} V_{T, m_{2}} \mathbf{1}_{\left\{S_{T}, m_{1} \geq K, V_{T, m_{2}<D *}\right\}}\right], \\
& A_{4}\left(m_{1}, m_{2}\right) \\
& \quad=-\frac{1-\alpha}{D} K E^{\mathrm{Q}_{\theta}}\left[V_{T, m_{2}} \mathbf{1}_{\left\{S_{T}, m_{1} \geq K, V_{T, m_{2}<D *}\right\}}\right] .
\end{aligned}
$$

Then, we can get $A_{1}\left(m_{1}, m_{2}\right), A_{2}\left(m_{1}, m_{2}\right), A_{3}\left(m_{1}, m_{2}\right)$, and $A_{4}\left(m_{1}, m_{2}\right)$ in closed form:

$$
\begin{aligned}
& A_{1}\left(m_{1}, m_{2}\right)=S_{0} e^{M_{S}\left(m_{1}\right)+(1 / 2)\left(\bar{U}_{1 T} T+m_{1} \sigma_{1}^{2}\right)} N_{2}\left(a_{1}\left(m_{1}\right),\right. \\
& \left.\quad a_{2}\left(m_{2}\right), \bar{\rho}\left(m_{1}, m_{2}\right)\right), \\
& A_{2}\left(m_{1}, m_{2}\right)=-K N_{2}\left(b_{1}\left(m_{1}\right), b_{2}\left(m_{2}\right), \bar{\rho}\left(m_{1}, m_{2}\right)\right), \\
& A_{3}\left(m_{1}, m_{2}\right)=\frac{1-\alpha}{D} \\
& \quad \cdot S_{0} V_{0} e^{M_{S}\left(m_{1}\right)+(1 / 2)\left(\bar{U}_{1 T} T+m_{1} \sigma_{1}^{2}\right)+M_{V}\left(m_{2}\right)+(1 / 2)\left(\bar{U}_{2 T} T+m_{2} \sigma_{2}^{2}\right)+\rho \bar{U}_{3 T} T} \\
& \quad \times N_{2}\left(c_{1}\left(m_{1}\right),\left(m_{2}\right),-\bar{\rho}\left(m_{1}, m_{2}\right)\right),
\end{aligned}
$$

$$
\begin{aligned}
& A_{4}\left(m_{1}, m_{2}\right)=-\frac{1-\alpha}{D} \\
& \cdot K V_{0} e^{M_{V}\left(m_{2}\right)+(1 / 2)\left(\bar{U}_{2 T} T+m_{2} \sigma_{2}^{2}\right)} N_{2}\left(d_{1}\left(m_{1}\right), d_{2}\left(m_{2}\right),\right. \\
& \left.\quad-\bar{\rho}\left(m_{1}, m_{2}\right)\right),
\end{aligned}
$$

where

$$
\begin{aligned}
& N_{2}\left(x_{1}, x_{2}, \rho\right) \\
& =\frac{1}{2 \pi \sqrt{1-\rho^{2}}} \int_{-\infty}^{x_{1}} \int_{-\infty}^{x_{2}} e^{-\left(1 / 2\left(1-\rho^{2}\right)\right)\left(p^{2}-2 \rho p q+q^{2}\right)} d p d q .
\end{aligned}
$$

The parameters are expressed as follows:

$$
\begin{aligned}
b_{1}\left(m_{1}\right)= & \frac{\ln \left(S_{0} / K\right)+M_{S}\left(m_{1}\right)}{\sqrt{\bar{U}_{1 T} T+m_{1} \sigma_{1}^{2}}}, \\
b_{2}\left(m_{2}\right)= & \frac{\ln \left(V_{0} / D^{*}\right)+M_{V}\left(m_{2}\right)}{\sqrt{\bar{U}_{2 T} T+m_{2} \sigma_{2}^{2}}}, \\
a_{1}\left(m_{1}\right)= & b_{1}\left(m_{1}\right)+\sqrt{\bar{U}_{1 T} T+m_{1} \sigma_{1}^{2}}, \\
a_{2}\left(m_{2}\right)= & b_{2}\left(m_{2}\right)+\bar{\rho} \sqrt{\bar{U}_{1 T} T+m_{1} \sigma_{1}^{2}}, \\
c_{1}\left(m_{1}\right)= & b_{1}\left(m_{1}\right) \\
& +\left(\sqrt{\bar{U}_{1 T} T+m_{1} \sigma_{1}^{2}}+\bar{\rho} \sqrt{\bar{U}_{2 T} T+m_{2} \sigma_{2}^{2}}\right), \\
c_{2}\left(m_{2}\right)= & -b_{2}\left(m_{2}\right) \\
& -\left(\sqrt{\bar{U}_{2 T} T+m_{2} \sigma_{2}^{2}}+\bar{\rho} \sqrt{\bar{U}_{1 T} T+m_{1} \sigma_{1}^{2}}\right), \\
d_{1}\left(m_{1}\right)= & b_{1}\left(m_{1}\right)+\bar{\rho} \sqrt{\bar{U}_{2 T} T+m_{2} \sigma_{2}^{2}}, \\
d_{2}\left(m_{2}\right)= & -b_{2}\left(m_{2}\right)-\sqrt{\bar{U}_{2 T} T+m_{2} \sigma_{2}^{2}} .
\end{aligned}
$$

Therefore, the conditional vulnerable European call option price given $\mathscr{F}_{T}^{U}$ is

$$
\begin{aligned}
C^{*} & =\sum_{n=0}^{\infty} \sum_{n_{1}=0}^{\infty} \sum_{n_{2}=0}^{\infty} Q\left(\widetilde{N}_{T}=n, N_{T}^{(1)}=n_{1}, N_{T}^{(2)}=n_{2}\right) C_{m_{1}, m_{2}} \\
& =\sum_{n=0}^{\infty} \sum_{n_{1}=0}^{\infty} \sum_{n_{2}=0}^{\infty} \frac{\left(\overline{\tilde{\lambda}}_{T} T\right)^{n}}{n !} \frac{\left(\bar{\lambda}_{S T} T\right)^{m_{1}-n}}{\left(m_{1}-n\right) !} \frac{\left(\bar{\lambda}_{V T} T\right)^{m_{2}-n}}{\left(m_{2}-n\right) !} e^{-\overline{\tilde{\lambda}}_{T} T-\bar{\lambda}_{S T} T-\bar{\lambda}_{V T} T} C_{m_{1}, m_{2}} .
\end{aligned}
$$

Note the valuation of vulnerable European call options depends on the parameters $\theta_{1 t}^{c_{1}}, \theta_{2 t}^{c_{2}}, \theta_{1 t-}^{J_{1}}$, and $\theta_{2 t-}^{J_{2}}$. For the numerical analysis, we use the special parameters that are given by Corollary 3 : 


$$
\begin{aligned}
M_{S}\left(m_{1}\right)= & E\left(\frac{\ln S_{T, m_{1}}}{\ln S_{0}}\right) \\
= & \int_{0}^{T}\left(r_{t}-k_{1} \lambda_{S t}-\frac{1}{2} \sigma_{S t}^{2}\right) d t+n_{1} \mu_{1} \\
& -\frac{1}{2} n \sigma_{1}^{2}, \\
M_{V}\left(m_{2}\right)= & E\left(\frac{\ln V_{T, m_{2}}}{\ln V_{0}}\right) \\
= & \int_{0}^{T}\left(r_{t}-k_{2} \lambda_{V t}-\frac{1}{2} \sigma_{V t}^{2}\right) d t+n_{2} \mu_{2} \\
& -\frac{1}{2} n \sigma_{2}^{2} .
\end{aligned}
$$

Note. If we adopt the hypothesis of Merton [9] and regard the jump risks as unsystematic risks which should not be priced, thus, the parameters describing the jumps in the two risky asset price processes will not be changed after application of the measure transform from the physical measure $P$ to risk-neutral measure. That is, the parameters $\theta_{1 t-}^{J_{1}}$ and $\theta_{2 t-}^{J_{2}}$ in (18) are equal to 0. From Theorem 1 and Proposition 2, we can obtain the results of the following two propositions specifically.

Proposition 4. The martingale conditions are satisfied if and only if

$$
\begin{aligned}
& \theta_{1 t}^{c_{1}}=\frac{\left(\beta_{t}-r_{t}\right) \rho \sigma_{S t}-\left(\alpha_{t}-r_{t}\right) \sigma_{V t}}{\left(1-\rho^{2}\right) \sigma_{S t}^{2} \sigma_{V t}}, \\
& \theta_{2 t}^{c_{2}}=\frac{\left(\alpha_{t}-r_{t}\right) \rho \sigma_{V t}-\left(\beta_{t}-r_{t}\right) \sigma_{S t}}{\left(1-\rho^{2}\right) \sigma_{V t}^{2} \sigma_{S t}} .
\end{aligned}
$$

Proposition 5. Conditional on $\mathscr{F}_{T}^{U}$ and under the equivalent martingale measure, the dynamics of $S_{t}$ and $V_{t}$ are given by

$$
\begin{aligned}
\frac{d S_{t}}{S_{t-}}= & \left(r_{t}-k_{1} \lambda_{S t}^{*}\right) d t+\sigma_{S t} d \widetilde{W}_{t}^{(1)} \\
& +\left(e^{Z_{S t-}^{(1)}}-1\right) d M_{t}^{(1)}, \\
\frac{d V_{t}}{V_{t-}}= & \left(r_{t}-k_{2} \lambda_{V t}^{*}\right) d t+\sigma_{V t} d \widetilde{W}_{t}^{(2)} \\
& +\left(e^{Z_{V t-}^{(1)}}-1\right) d M_{t}^{(2)},
\end{aligned}
$$

where $\widetilde{W}_{t}^{(1)}$ and $\widetilde{W}_{t}^{(2)}$ are two standard Brownian motions which is defined by (31).

Under the conditions $\mathbb{G}_{T}^{\left(n, n_{1}, n_{2}\right)}:=\left\{N_{T}=n, N_{T}^{(1)}=\right.$ $\left.n_{1}, N_{T}^{(2)}=n_{2}\right\}$ and $\mathscr{F}_{T}^{U}$, for $m_{1}=n+n_{1}, m_{2}=n+$ $n_{2},\left(\ln S_{T, m_{1}} / \ln S_{0}, \ln V_{T, m_{2}} / \ln V_{0}\right)$ is also bivariate normally distributed with the following properties:

$$
\begin{gathered}
M_{S}^{\prime}\left(m_{1}\right)=E\left(\frac{\ln S_{T, m_{1}}}{\ln S_{0}}\right) \\
=\int_{0}^{T}\left(r_{t}-k_{1} \lambda_{S t}^{*}-\frac{1}{2} \sigma_{S t}^{2}\right) d t+m_{1} \mu_{1}, \\
M_{V}^{\prime}\left(m_{2}\right)=E\left(\frac{\ln V_{T, m_{2}}}{\ln V_{0}}\right) \\
=\int_{0}^{T}\left(r_{t}-k_{2} \lambda_{V t}^{*}-\frac{1}{2} \sigma_{V t}^{2}\right) d t+m_{2} \mu_{2}, \\
\operatorname{cov}\left(\frac{\ln S_{T, m_{1}}}{\ln S_{0}}, \frac{\ln V_{T, m_{2}}}{\ln V_{0}}\right)=\int_{0}^{T} \rho \sigma_{S t} \sigma_{V t} d t .
\end{gathered}
$$

Similar to the previous results, we can obtain

$$
\begin{aligned}
C^{*} & =\sum_{n=0}^{\infty} \sum_{n_{1}=0}^{\infty} \sum_{n_{2}=0}^{\infty} Q\left(N_{T}=n, N_{T}^{(1)}=n_{1}, N_{T}^{(2)}=n_{2}\right) C_{m_{1}, m_{2}}^{\prime} \\
& =\sum_{n=0}^{\infty} \sum_{n_{1}=0}^{\infty} \sum_{n_{2}=0}^{\infty} \frac{\left(\bar{\lambda}_{T} T\right)^{n}}{n !} \frac{\left(\bar{\lambda}_{S T} T\right)^{m_{1}-n}}{\left(m_{1}-n\right) !} \frac{\left(\bar{\lambda}_{V T} T\right)^{m_{2}-n}}{\left(m_{2}-n\right) !} e^{-\bar{\lambda}_{T} T-\bar{\lambda}_{S T} T-\bar{\lambda}_{V T} T} C_{m_{1}, m_{2}}^{\prime},
\end{aligned}
$$

where $\bar{\lambda}_{T}=(1 / T) \int_{0}^{T} \lambda_{t} d t=(1 / T) \sum_{i=1}^{N} \lambda_{i} J_{i}$. So

$$
\begin{aligned}
& C_{m_{1}, m_{2}}^{\prime}=e^{-\bar{R}_{T} T}\left[A_{1}^{\prime}\left(m_{1}, m_{2}\right)+A_{2}^{\prime}\left(m_{1}, m_{2}\right)\right. \\
& \left.\quad+A_{3}^{\prime}\left(m_{1}, m_{2}\right)+A_{4}^{\prime}\left(m_{1}, m_{2}\right)\right] .
\end{aligned}
$$

The forms of $A_{1}^{\prime}\left(m_{1}, m_{2}\right), A_{2}^{\prime}\left(m_{1}, m_{2}\right), A_{3}^{\prime}\left(m_{1}, m_{2}\right)$, and $A_{4}^{\prime}\left(m_{1}, m_{2}\right)$ are consistent with those given in (50), as long as $M_{S}\left(m_{1}\right)$ is replaced with $M_{S}^{\prime}\left(m_{1}\right)$ and $M_{V}\left(m_{2}\right)$ is replaced with $M_{V}^{\prime}\left(m_{2}\right)$.

\section{Vulnerable European Option Pricing Considering the Market Prices of All Jump Risks}

For comparison, we can follow Bo et al. [15] and assume that all jump risks are systematic risks which should be priced. 
Therefore, the parameters describing the jumps in the two risky asset value processes should be changed. As in (15), we still define two processes $Y_{1 t}$ and $Y_{2 t}$. For simplicity, let

$$
\begin{aligned}
& Y_{1 t}=C_{1 t}+J_{1 t}+J_{t}, \\
& Y_{2 t}=C_{2 t}+J_{2 t}+J_{t}^{\prime},
\end{aligned}
$$

and they admit the following forms:

$$
\begin{aligned}
C_{1 t} & =\int_{0}^{t}\left(\alpha_{s}-k_{1} \lambda_{S s}^{*}-\frac{1}{2} \sigma_{S s}^{2}\right) d s+\int_{0}^{t} \sigma_{S s} d W_{s}^{(1)}, \\
J_{1 t} & =\int_{0}^{t} Z_{S s^{-}}^{(1)} d N_{s}^{(1)}, \\
J_{t} & =\int_{0}^{t} Z_{S s^{-}}^{(1)} d N_{s}, \\
C_{2 t} & =\int_{0}^{t}\left(\beta_{s}-k_{2} \lambda_{V s}^{*}-\frac{1}{2} \sigma_{V s}^{2}\right) d s+\int_{0}^{t} \sigma_{V s} d W_{s}^{(2)}, \\
J_{2 t} & =\int_{0}^{t} Z_{V s-}^{(2)} d N_{s}^{(2)}, \\
J_{t}^{\prime} & =\int_{0}^{t} Z_{V s-}^{(2)} d N_{s} .
\end{aligned}
$$

Because the Brownian motions and jump processes are independent, the regime-switching Esscher transforms for diffusion processes and jump processes are defined separately as follows.

We adopt the Esscher transform for the diffusion parts to determine an equivalent martingale measure.

$$
\begin{aligned}
& \left.\frac{d Q_{\theta_{c}}}{d P}\right|_{\mathscr{H}_{T}} \\
& =\frac{\exp \left(\int_{0}^{T} \theta_{1 t}^{c_{1}} d C_{1 t}+\int_{0}^{T} \theta_{2 t}^{c_{2}} d C_{2 t}\right)}{E\left[\exp \left(\int_{0}^{T} \theta_{1 t}^{c_{1}} d C_{1 t}+\int_{0}^{T} \theta_{2 t}^{c_{2}} d C_{2 t}\right) \mid \mathscr{F}_{T}^{U}\right]},
\end{aligned}
$$

where $E[\cdot]$ is the expectation under measure P. $\theta_{1 t}^{c_{1}}=\left\langle\theta_{1}^{c_{1}}, U_{t}\right\rangle$, $\theta_{1}^{c_{1}}=\left(\theta_{11}^{c_{1}}, \theta_{12}^{c_{1}}, \ldots, \theta_{1 N}^{c_{1}}\right) \in \mathbf{R}^{N} ; \theta_{2 t}^{c_{2}}=\left\langle\theta_{2}^{c_{2}}, U_{t}\right\rangle, \theta_{2}^{c_{2}}=$ $\left(\theta_{21}^{c_{2}}, \theta_{22}^{c_{2}}, \ldots, \theta_{2 N}^{c_{2}}\right) \in \mathbf{R}^{N}$. It is easy to obtain

$$
\begin{aligned}
& \left.\frac{d Q_{\theta_{c}}}{d P}\right|_{\mathscr{H}_{T}}=\exp \left(\int_{0}^{T} \theta_{1 t}^{c_{1}} \sigma_{S t} d W_{t}^{(1)}+\int_{0}^{T} \theta_{2 t}^{c_{2}} \sigma_{V t} d W_{t}^{(2)}\right. \\
& -\frac{1}{2} \\
& \left.\cdot \int_{0}^{T}\left(\left(\theta_{1 t}^{c_{1}}\right)^{2} \sigma_{S t}^{2}+\left(\theta_{2 t}^{c_{2}}\right)^{2} \sigma_{V t}^{2}+2 \rho \theta_{1 t}^{c_{1}} \sigma_{S t} \theta_{2 t}^{c_{2}} \sigma_{V t}\right) d t\right) .
\end{aligned}
$$

The Esscher transform for the jump processes to determine an equivalent martingale measure is given below:

$$
\left.\frac{d Q_{\theta_{J}}}{d P}\right|_{\mathscr{H}_{T}}=\frac{\exp \left(\int_{0}^{T} \theta_{1 t}^{J_{1}} d J_{1 t}+\int_{0}^{T} \theta_{t}^{J} d J_{t}+\int_{0}^{T} \theta_{2 t}^{I_{2}} d J_{2 t}+\int_{0}^{\mathrm{T}} \theta_{t}^{J^{\prime}} d J_{t}^{\prime}\right)}{E\left[\exp \left(\int_{0}^{T} \theta_{1 t}^{I_{1}} d J_{1 t}+\int_{0}^{T} \theta_{t}^{J} d J_{t}+\int_{0}^{T} \theta_{2 t}^{I_{2}} d J_{2 t}+\int_{0}^{T} \theta_{t}^{J^{\prime}} d J_{t}^{\prime}\right) \mid \mathscr{F}_{T}^{U}\right]},
$$

from which we can obtain

$$
\begin{aligned}
& \left.\frac{d Q_{\theta_{I}}}{d P}\right|_{\mathscr{H}_{T}}=\exp \left(\int_{0}^{T} \theta_{1 t}^{J_{1}} Z_{S t-}^{(1)} d N_{t}^{(1)}\right. \\
& \left.-\int_{0}^{T} \lambda_{S t}\left(e^{\theta_{1 t}^{J_{1}} \mu_{1}+(1 / 2)\left(\theta_{1 t}^{J_{1}} \sigma_{1}\right)^{2}}-1\right) d t\right) \\
& \quad \times \exp \left(\int_{0}^{T} \theta_{2 t}^{J_{2}} Z_{V t}^{(2)} d N_{t}^{(2)}\right. \\
& \left.-\int_{0}^{T} \lambda_{V t}\left(e^{\theta_{2 t}^{J_{2}} \mu_{2}+(1 / 2)\left(\theta_{2 t}^{I_{2}} \sigma_{2}\right)^{2}}-1\right) d t\right) \\
& \quad \times \exp \left(\int_{0}^{T}\left(\theta_{t}^{J} Z_{S t}^{(1)}+\theta_{t}^{J^{\prime}} Z_{V t}^{(2)}\right) d N_{t}\right. \\
& \left.-\int_{0}^{T} \lambda_{t}\left(e^{\left(\theta_{t}^{J} \mu_{1}+\theta_{t}^{J^{\prime}} \mu_{2}\right)+(1 / 2)\left(\left(\theta_{t}^{J} \sigma_{1}\right)^{2}+\left(\theta_{t}^{J^{\prime}} \sigma_{2}\right)^{2}\right)}-1\right) d t\right) .
\end{aligned}
$$

Based on the above analysis, we now define a new probability measure $Q_{\theta}^{\prime}$ by setting

$$
\left.\frac{d Q_{\theta}^{\prime}}{d P}\right|_{\mathscr{H}_{T}}=\left.\frac{d Q_{\theta_{I}}}{d P}\right|_{\mathscr{H}_{T}} \times\left.\frac{d Q_{\theta_{c}}}{d P}\right|_{\mathscr{H}_{T}} .
$$

Theorem 6. The martingale conditions are satisfied if and only if

$$
\begin{aligned}
& \alpha_{t}-r_{t}-k_{1} \lambda_{S t}^{*}+\theta_{1 t}^{c_{1}} \sigma_{S t}^{2}+\rho \sigma_{S t} \theta_{2 t}^{c_{2}} \sigma_{V t}+\tilde{\lambda}_{S t} k_{S t}^{\theta_{1 t}^{J_{1}}} \\
& +\tilde{\lambda}_{t} k_{t}^{\theta_{t}^{J}}=0, \\
& \beta_{t}-r_{t}-k_{2} \lambda_{V t}^{*}+\theta_{2 t}^{c_{2}} \sigma_{V t}^{2}+\rho \sigma_{S t} \theta_{1 t}^{c_{1}} \sigma_{V t}+\tilde{\lambda}_{V t} k_{V t}^{\theta_{2 t}^{J_{2}}} \\
& +\tilde{\lambda}_{t} k_{t}^{\theta_{t}^{J^{\prime}}}=0,
\end{aligned}
$$

where

$$
\begin{aligned}
\tilde{\lambda}_{t} & =\lambda_{t} e^{\left(\theta_{t}^{J} \mu_{1}+\theta_{t}^{I^{\prime}} \mu_{2}\right)+(1 / 2)\left(\left(\theta_{t}^{J} \sigma_{1}\right)^{2}+\left(\theta_{t}^{J^{\prime}} \sigma_{2}\right)^{2}\right)}, \\
\tilde{\lambda}_{S t} & =\lambda_{S t} e^{\theta_{1 t}^{I_{1}} \mu_{1}+(1 / 2)\left(\theta_{1 t}^{J_{1}} \sigma_{1}\right)^{2}}, \\
\tilde{\lambda}_{V t} & =\lambda_{V t} e^{\theta_{2 t}^{J_{2}} \mu_{2}+(1 / 2)\left(\theta_{2 t}^{J_{2}} \sigma_{2}\right)^{2}}, \\
k_{t}^{\theta_{t}^{J}} & =\left(k_{1}+1\right) e^{\theta_{t}^{J} \sigma_{1}^{2}}-1,
\end{aligned}
$$




$$
\begin{aligned}
& k_{t}^{\theta_{t}^{J^{\prime}}}=\left(k_{2}+1\right) e^{\theta_{t}^{J^{\prime}} \sigma_{2}^{2}}-1, \\
& k_{S t}^{\theta_{t 1}^{J_{1}}}=\left(k_{1}+1\right) e^{\theta_{1 t}^{I_{1}} \sigma_{1}^{2}}-1, \\
& k_{V t}^{\theta_{t 2}^{I_{2}}}=\left(k_{2}+1\right) e^{\theta_{2 t}^{I_{2}} \sigma_{2}^{2}}-1 .
\end{aligned}
$$

The proof is the same as for Theorem 1 in Section 3, and we omit it here.

Proposition 7. Conditional on $\mathscr{F}_{T}^{U}$ and under the equivalent martingale measure $Q_{\theta}^{\prime}$, one has

$$
\begin{aligned}
\ln S_{T}= & \ln S_{0}+\int_{0}^{T}\left(r_{t}-\widetilde{\lambda}_{S t} k_{S t}^{\theta_{1 t}^{J_{1}}}-\widetilde{\lambda}_{t} k_{t}^{\theta_{t}^{I}}-\frac{1}{2} \sigma_{S t}^{2}\right) d t \\
& +\int_{0}^{T} \sigma_{S t} d \widetilde{W}_{t}^{(1)}+\sum_{i=1}^{\widetilde{N}_{T}^{(1)}} \widetilde{Z}_{S i}^{(1)}+\sum_{i=1}^{\widetilde{N}_{T}} \widehat{Z}_{S i}^{(1)}, \\
\ln V_{T}= & \ln V_{0}+\int_{0}^{T}\left(r_{t}-\widetilde{\lambda}_{V t} k_{V t}^{\theta_{2 t}^{J_{2}}}-\widetilde{\lambda}_{t} k_{t}^{\theta_{t}^{I^{\prime}}}-\frac{1}{2} \sigma_{V t}^{2}\right) d t \\
& +\int_{0}^{T} \sigma_{V t} d \widetilde{W}_{t}^{(2)}+\sum_{i=1}^{\widetilde{N}_{T}^{(2)}} \widetilde{Z}_{V i}^{(2)}+\sum_{i=1}^{\widetilde{N}_{T}} \widehat{Z}_{V i}^{(2)},
\end{aligned}
$$

where $\widetilde{W}_{t}^{(1)}$ and $\widetilde{W}_{t}^{(2)}$ are also two standard Brownian motions following (31).

Then, all the parameters describing the jumps in the two risky asset values are changed according to (70). The probability law of the chain $U$ remains the same after the measure change; that is, under $Q_{\theta}^{\prime}, U$ still has the semimartingale dynamics.

Moreover, because the solutions of (69) are not unique for each $0 \leq t \leq T$, we obtain a special pair of solutions by making some assumptions. Let

$$
\begin{aligned}
\alpha_{t}-r_{t}-k_{1} \lambda_{S t}^{*}+\theta_{1 t}^{c_{1}} \sigma_{S t}^{2}+\rho \sigma_{S t} \theta_{2 t}^{c_{2}} \sigma_{V t} & =0, \\
\tilde{\lambda}_{S t}\left(e^{\mu_{1}+(1 / 2) \sigma_{1}^{2}+\theta_{1 t}^{J_{1}} \sigma_{1}^{2}}-1\right) & =0, \\
\tilde{\lambda}_{t}\left(e^{\mu_{1}+(1 / 2) \sigma_{1}^{2}+\theta_{t}^{I} \sigma_{1}^{2}}-1\right) & =0, \\
\beta_{t}-r_{t}-k_{2} \lambda_{V t}^{*}+\theta_{t 2}^{c_{2}} \sigma_{V t}^{2}+\rho \sigma_{S t} \theta_{1 t}^{c_{1}} \sigma_{V t} & =0, \\
\tilde{\lambda}_{V t}\left(e^{\mu_{2}+(1 / 2) \sigma_{2}^{2}+\theta_{2 t}^{I_{2}} \sigma_{2}^{2}}-1\right) & =0, \\
\tilde{\lambda}_{t}\left(e^{\mu_{2}+(1 / 2) \sigma_{2}^{2}+\theta_{t}^{J^{\prime}} \sigma_{2}^{2}}-1\right) & =0,
\end{aligned}
$$

then we can have the following result.
Corollary 8. Under the assumptions of (72), the martingale conditions can be satisfied if the parameters are given as follows:

$$
\begin{aligned}
& \theta_{1 t}^{c_{1} *}=\frac{\left(\beta_{t}-r_{t}-k_{2} \lambda_{V t}^{*}\right) \rho \sigma_{S t}-\left(\alpha_{t}-r_{t}-k_{1} \lambda_{S t}^{*}\right) \sigma_{V t}}{\left(1-\rho^{2}\right) \sigma_{S t}^{2} \sigma_{V t}}, \\
& \theta_{2 t}^{c_{2}^{*}}=\frac{\left(\alpha_{t}-r_{t}-k_{1} \lambda_{S t}^{*}\right) \rho \sigma_{V t}-\left(\beta_{t}-r_{t}-k_{2} \lambda_{V t}^{*}\right) \sigma_{S t}}{\left(1-\rho^{2}\right) \sigma_{V t}^{2} \sigma_{S t}}, \\
& \theta_{1 t}^{J_{1}^{*}}=\theta_{t}^{J^{*}}=-\frac{\mu_{1}+(1 / 2) \sigma_{1}^{2}}{\sigma_{1}^{2}}, \\
& \theta_{2 t}^{J_{2}^{*}}=\theta_{t}^{J^{\prime *}}=-\frac{\mu_{2}+(1 / 2) \sigma_{2}^{2}}{\sigma_{2}^{2}} \\
& \tilde{k}_{S t}^{\theta_{1 t}^{J_{1}}}=\widetilde{k}_{t}^{\theta_{t}^{J}}=\left(k_{1}+1\right) e^{\theta_{1 t}^{J_{1}}{ }^{*} \sigma_{1}^{2}}-1=0, \\
& \tilde{k}_{V t}^{\theta_{2 t}^{I_{2}}}=\widetilde{k}_{t}^{\theta_{t}^{J^{\prime}}}=\left(k_{2}+1\right) e^{\theta_{2 t}^{J_{2}{ }^{*}} \sigma_{2}^{2}}-1=0, \\
& \tilde{\lambda}_{t}^{*}=\lambda_{t} \exp \left(-\frac{\mu_{1}^{2}}{2 \sigma_{1}^{2}}+\frac{\sigma_{1}^{2}}{8}\right) \times \exp \left(-\frac{\mu_{2}^{2}}{2 \sigma_{2}^{2}}+\frac{\sigma_{2}^{2}}{8}\right), \\
& \tilde{\lambda}_{S t}^{*}=\lambda_{S t} \exp \left(-\frac{\mu_{1}^{2}}{2 \sigma_{1}^{2}}+\frac{\sigma_{1}^{2}}{8}\right), \\
& \tilde{\lambda}_{V t}^{*}=\lambda_{V t} \exp \left(-\frac{\mu_{2}^{2}}{2 \sigma_{2}^{2}}+\frac{\sigma_{2}^{2}}{8}\right) \text {. }
\end{aligned}
$$

The explicit Esscher parameters in Corollary 8 are used into numerical analysis. Consequently, we have the dynamics of the asset value processes under the equivalent martingale measure $Q_{\theta}^{\prime}$ with the Esscher parameters in Corollary 8. Under the conditions $\mathbb{G}_{T}^{\left(n, n_{1}, n_{2}\right)}:=\left\{\widetilde{N}_{T}=n, \widetilde{N}_{T}^{(1)}=\right.$ $\left.n_{1}, \widetilde{N}_{T}^{(2)}=n_{2}\right\}$ and $\mathscr{F}_{T}^{U}$, for $m_{1}=n+n_{1}, m_{2}=n+$ $n_{2},\left(\ln S_{T, m_{1}} / \ln S_{0}, \ln V_{T, m_{2}} / \ln V_{0}\right)$ is also bivariate normally distributed with the following properties:

$$
\begin{aligned}
& M_{S}^{\prime \prime}\left(m_{1}\right)=E\left(\frac{\ln S_{T, m_{1}}}{\ln S_{0}}\right) \\
& =\int_{0}^{T}\left(r_{t}-\frac{1}{2} \sigma_{S t}^{2}\right) d t-\frac{1}{2} m_{1} \sigma_{1}^{2}, \\
& M_{V}^{\prime \prime}\left(m_{2}\right)=E\left(\frac{\ln V_{T, m_{2}}}{\ln V_{0}}\right) \\
& =\int_{0}^{T}\left(r_{t}-\frac{1}{2} \sigma_{V t}^{2}\right) d t-\frac{1}{2} m_{2} \sigma_{2}^{2}, \\
& \operatorname{cov}\left(\frac{\ln S_{T, m_{1}}}{\ln S_{0}}, \frac{\ln V_{T, m_{2}}}{\ln V_{0}}\right)=\int_{0}^{T} \rho \sigma_{S t} \sigma_{V t} d t .
\end{aligned}
$$




$$
\begin{aligned}
C^{*} & =\sum_{n=0}^{\infty} \sum_{n_{1}=0}^{\infty} \sum_{n_{2}=0}^{\infty} Q\left(\widetilde{N}_{T}=n, \widetilde{N}_{T}^{(1)}=n_{1}, \widetilde{N}_{T}^{(2)}=n_{2}\right) C_{m_{1}, m_{2}}^{\prime \prime} \\
& =\sum_{n=0}^{\infty} \sum_{n_{1}=0}^{\infty} \sum_{n_{2}=0}^{\infty} \frac{\left(\overline{\widetilde{\lambda}}_{T} T\right)^{n}}{n !} \frac{\left(\overline{\widetilde{\lambda}}_{S T} T\right)^{m_{1}-n}}{\left(m_{1}-n\right) !} \frac{\left(\overline{\widetilde{\lambda}}_{V T} T\right)^{m_{2}-n}}{\left(m_{2}-n\right) !} e^{-\overline{\tilde{\lambda}}_{T} T-\overline{\tilde{\lambda}}_{S T} T-\overline{\tilde{\lambda}}_{V T} T} C_{m_{1}, m_{2}}^{\prime \prime},
\end{aligned}
$$

where

$$
\begin{aligned}
& \overline{\tilde{\lambda}}_{S T}=\frac{1}{T} \int_{0}^{T} \tilde{\lambda}_{S t} d t=\frac{1}{T} \sum_{i=1}^{N} \widetilde{\lambda}_{S t_{i}} J_{i} \\
& \overline{\widetilde{\lambda}}_{V T}=\frac{1}{T} \int_{0}^{T} \tilde{\lambda}_{V t} d t=\frac{1}{T} \sum_{i=1}^{N} \widetilde{\lambda}_{V t_{i}} J_{i} .
\end{aligned}
$$

So

$$
\begin{aligned}
& C_{m_{1}, m_{2}}^{\prime \prime}=e^{-\bar{R}_{T} T}\left[A_{1}^{\prime \prime}\left(m_{1}, m_{2}\right)+A_{2}^{\prime \prime}\left(m_{1}, m_{2}\right)\right. \\
& \left.+A_{3}^{\prime \prime}\left(m_{1}, m_{2}\right)+A_{4}^{\prime \prime}\left(m_{1}, m_{2}\right)\right],
\end{aligned}
$$

and the forms of $A_{1}^{\prime \prime}\left(m_{1}, m_{2}\right), A_{2}^{\prime \prime}\left(m_{1}, m_{2}\right), A_{3}^{\prime \prime}\left(m_{1}, m_{2}\right)$, and $A_{4}^{\prime \prime}\left(m_{1}, m_{2}\right)$ are consistent with those given in Section 3 , as long as $M_{S}\left(m_{1}\right)$ is replaced with $M_{S}^{\prime \prime}\left(m_{1}\right)$ and $M_{V}\left(m_{2}\right)$ is replaced with $M_{V}^{\prime \prime}\left(m_{2}\right)$.

\section{Numerical Results}

In this section, we adopt Monte Carlo simulation to preform numerical experiments for the vulnerable European call option prices under the regime-switching jump-diffusion models. We first present numerical results for the vulnerable European call option prices considering the market prices of common systematic jump risks. The effects of some basic variables on vulnerable option prices which are obtained from formula (55) with the Esscher parameters in Corollary 3 are illustrated in Figures 1-4. Suppose one year has 252 trading days. Then, the sample interval is $1 / 252$. We parsimoniously assume that the Markov chain $U$ has two states; that is, $N=2$. The first and second regimes, namely, $e_{1}=(1,0)^{\prime}$ and $e_{2}=(0,1)^{\prime}$, can be interpreted as good and bad economic states, respectively. We suppose that the transition probability matrix $Q$ of the two-state Markov chain $U$ is given by

$$
\left(\begin{array}{ll}
p_{11} & p_{12} \\
p_{21} & p_{22}
\end{array}\right)=\left(\begin{array}{ll}
0.2 & 0.8 \\
0.7 & 0.3
\end{array}\right) \text {. }
$$

We choose these parameters to be broadly consistent with those used in the literature such as Tian et al. [12] to calibrate the results. The preference parameters listed in Table 1 are independent of the Markov chain, but the parameters listed in Table 2 depend on the Markov chain. We suppose that the initial state of the economy be $e_{1}$. In Figures $1-6$, we change one of the parameter values to investigate its impact on the vulnerable option price, with the other variables taking on the values listed in Tables 1 and 2.

Figure 1 shows the vulnerable European call option price plotted against the spot-to-strike ratio for three different cases. The "+", “०”, and “*” lines correspond to the cases of mixed states with transition probability matrix $Q$, pure state 1 , and pure state 2 , respectively. It can be seen that the option values when considering the mixed states of the economy are higher than the values in pure state 1 (good state) and lower than the values in pure state 2 (bad state). Mixed states of the economy can better consider the influence of a change in the economic system on the option value. If the model considers only state 1 and ignores the regime-switching and jump risk, the numerical results are the same as the result in Figure 3 in Tian et al. [12].

Three-dimensional numerical analysis of the impact of the initial prices of $S\left(S_{0}\right)$ and $V\left(V_{0}\right)$ on the vulnerable European call option price is performed. We can see that the option price increases with the increase in $S_{0}$ and $V_{0}$, but the impact of $S_{0}$ on the option value is stronger than that of $V_{0}$.

Figure 3 shows the effects of the mean jump sizes $\mu_{1}$ of $S_{t}$ and $\mu_{2}$ of $V_{t}$ on the option prices under mixed states. If $\mu_{2}=0$ remains unchanged, the vulnerable option price traces out a U-shaped curve as $\mu_{1}$ change from -1 to 1 . If $\mu_{1}=0$ remains unchanged, the option value presents an inverse $U$ shaped curve when $\mu_{2}$ changes from -1 to 1 . By comparing the curvature degrees, we can illustrate that the impact of $\mu_{1}$ on the option value is stronger than that of $\mu_{2}$. Compared to Figure 12 in Tian et al. [12], the option value curves are of similar shape, but the values are distinct.

Figure 4 shows the effects of the standard deviations of the jump sizes $\sigma_{1}$ of $S$ and $\sigma_{2}$ of $V$ on the option prices under mixed states. If $\sigma_{2}=0.1$ remains unchanged, the option value presents an increasing trend as $\sigma_{1}$ changes from 0 to 0.8 , while if $\sigma_{1}=0.1$ remains unchanged, the option value declines as $\sigma_{2}$ changes from 0 to 0.8 . We can see from the rates of change that the influence of $\sigma_{1}$ is more prominent. The conclusion is similar to that of Figure 13 in Tian et al. [12]. Figures 3 and 4 indicate that the impacts of the mean jump size and the standard deviation of the jump size of the underlying asset on the option price are more significant than that of the assets of the counterparty.

Below, we consider the option prices against the parameters for three cases of incorporating the market prices of jump risks in Figures 5-6. The “o”, “+”, and “*” lines correspond 
TABLE 1: Parameters that are independent of the Markov chain.

\begin{tabular}{lc}
\hline Parameter name & Value \\
\hline Mean jump size of $S$ & $\mu_{1}=0$ \\
Volatility of jump size of $S$ & $\sigma_{1}=0.1$ \\
Initial value of $S$ & $S_{0}=10$ \\
Correlation coefficient $\rho$ & $\rho=0.5$ \\
Default boundary & $D^{*}=10$ \\
Time to maturity & $T=1$ \\
Mean jump size of $V$ & $\mu_{2}=0$ \\
Volatility of jump size of $V$ & $\sigma_{2}=0.1$ \\
Initial value of $V$ & $V_{0}=10$ \\
Strike price & $K=10$ \\
Outstanding claims & $D=10$ \\
Deadweight cost of bankruptcy & $\alpha=0.5$ \\
\hline
\end{tabular}

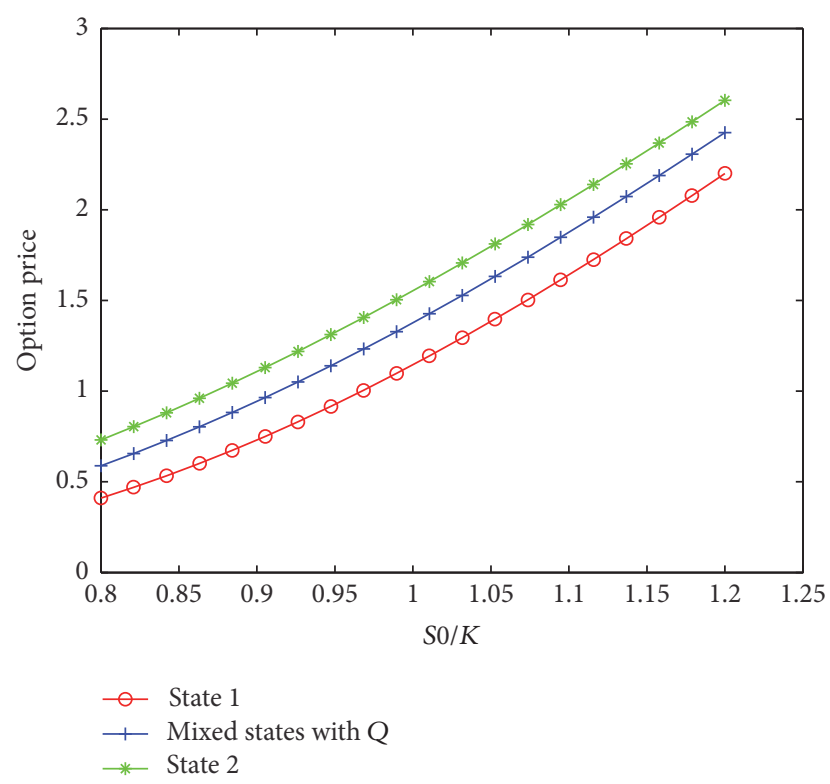

FIGURE 1: Vulnerable European call option price against spot-tostrike ratio.

to the cases of considering the market prices of common jump risks obtained from formula (55) with the Esscher parameters in Corollary 3, neglecting the market prices of all jump risks obtained from formula (60) and considering the market prices of all jump risks obtained from formula (75) with the Esscher parameters in Corollary 8, respectively. Figure 5 shows that the difference in vulnerable European call option prices is small against the spot-to-strike ratio for three cases with parameters $\mu_{1}=0, \mu_{2}=0, \sigma_{1}=0.1, \sigma_{2}=0.1$ and other parameters taking on the values listed in Tables 1 and 2. The numerical results are inconsistent with what I had expected that incorporating the market prices of jump risks would increase the option prices. One of the reasons may be the choice of special regime-switching parameters for the equivalent martingale measure. This problem needs to be researched. To investigate whether the choice of model parameters has an impact on the results, we change the

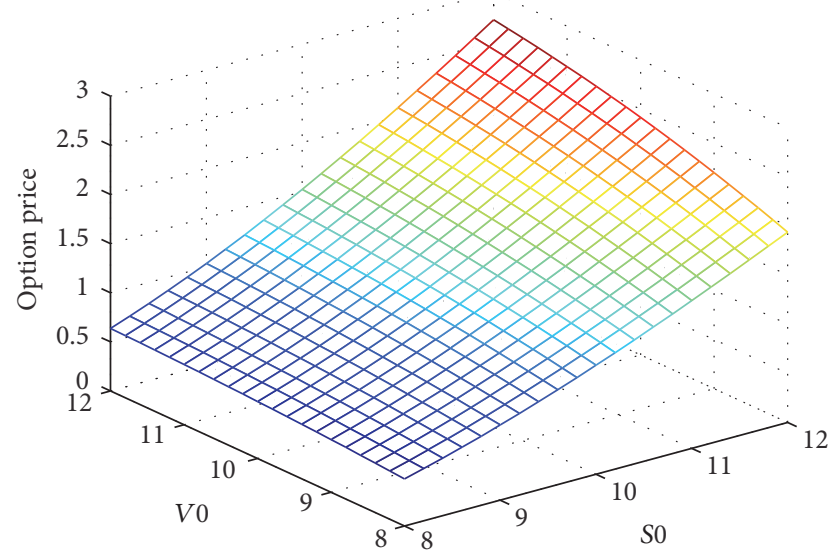

FIGURE 2: Vulnerable European call option price against the initial prices of $S$ and $V$.

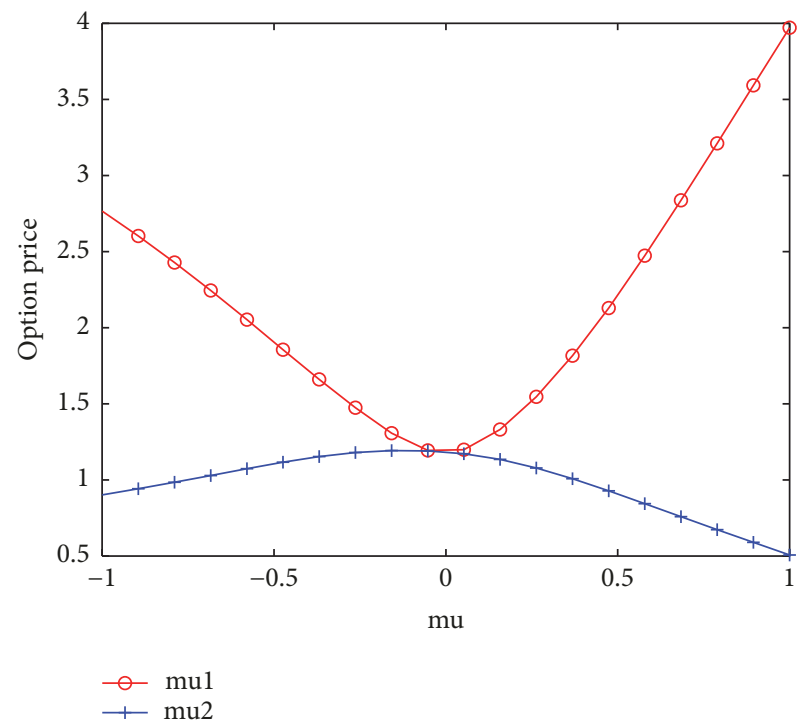

FIgURE 3: Vulnerable European call option price against mean jump sizes of $S$ and $V$.

parameters to $\mu_{1}=0.01, \mu_{2}=0.09, \sigma_{1}=0.1, \sigma_{2}=0.3$. From Figure 6 , we can see clearly that, with gradual incorporation of the market prices of jump risks, the option prices increase against the spot-to-strike ratio. Moreover, with the increase in $S_{0} / K$, the differences in the option prices between the three cases increase. Thus, we can see that the introduction of the market prices of jump risks affects the option value and that the option price when considering the market prices of common jump risks is between the option prices in the other two cases. Therefore, studying this problem has practical significance. Note that the effects of the other parameters of the models can also be discussed.

\section{Conclusion}

This paper proposes the pricing problem of vulnerable European options, in which the dynamics of the underlying 
TABle 2: Parameters that depend on the Markov chain.

\begin{tabular}{lcc}
\hline Parameter name & Value in state $e_{1}$ & Value in state $e_{2}$ \\
\hline Interest rate & $r_{1}=0.02$ & $r_{2}=0.04$ \\
Volatility of $S$ & $\sigma_{S 1}=0.3$ & $\sigma_{S 2}=0.4$ \\
Volatility of $V$ & $\sigma_{V 1}=0.3$ & $\sigma_{V 2}=0.4$ \\
Common jump intensity & $\lambda_{1}=1$ & $\lambda_{2}=2$ \\
Individual jump intensity of $S$ & $\lambda_{S 1}=1$ & $\lambda_{S 2}=2$ \\
Individual jump intensity of $V$ & $\lambda_{V 1}=1$ & $\lambda_{V 1}=2$ \\
\hline
\end{tabular}

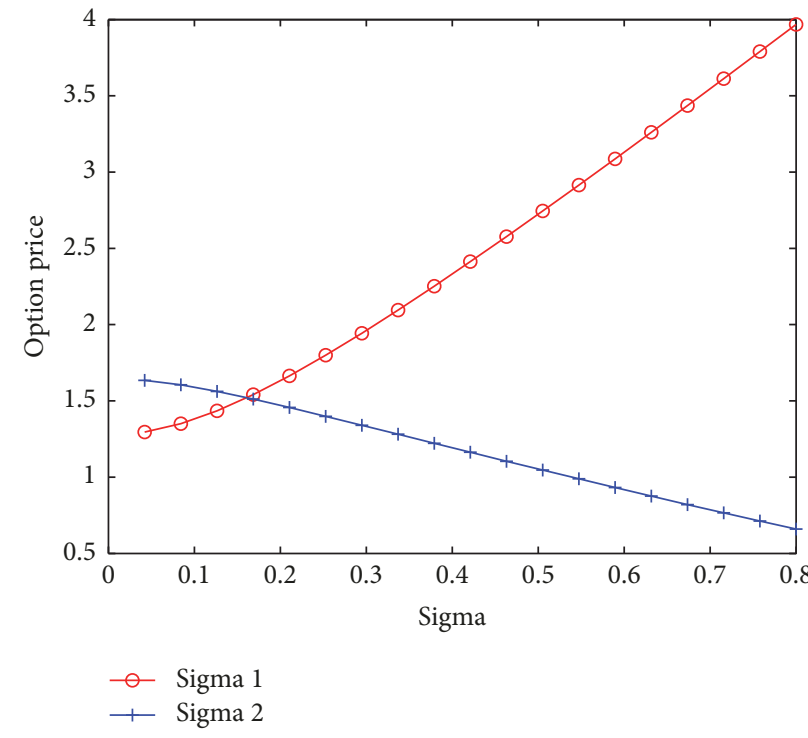

FIGURE 4: Vulnerable European call option price against standard deviation of the jump sizes of $S$ and $V$.

asset of the option and the asset value of the counterparty are driven by two correlated Markov regime-switching jumpdiffusion processes. Comparing with most of the existing regime-switching jump-diffusion models, the main advantage of our model is that we incorporate the market prices of common jump risks as systematic risks into the models because the jump processes are divided into individual jumps for each asset price and common jumps that affect the prices of all assets. We employ the Esscher transform to determine an equivalent martingale measure in the incomplete market and obtain the vulnerable options pricing formulae. To illustrate the influence of the market prices of jump risks on the price of vulnerable options, vulnerable options pricing formulae are also given in the case of taking and without taking the market prices of all jump risks into consideration. The numerical results indicate that jump risks have a more significant impact on the option prices. As potential future works, we might consider incorporating regime-switching risk into options pricing and discuss the effect of the choice of regime-switching Esscher parameters on the option prices.

\section{Conflicts of Interest}

There are no conflicts of interest related to this paper.

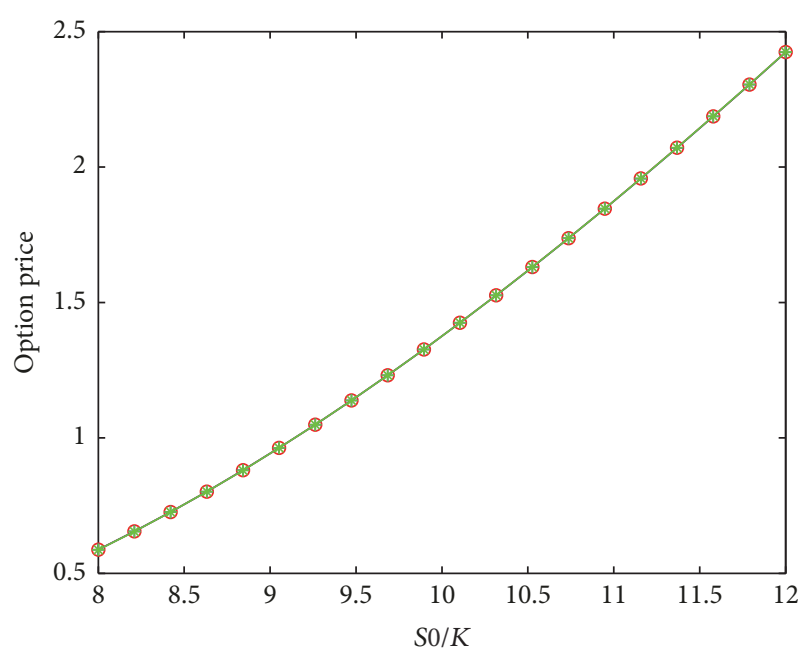

FIGURE 5: Vulnerable European call option price against spot-tostrike ratio for three cases with parameters $\mu_{1}=0, \mu_{2}=0, \sigma_{1}=0.1$, and $\sigma_{2}=0.1$.



FIGURE 6: Vulnerable European call option price against spot-tostrike ratio for three cases with parameters $\mu_{1}=0.01, \mu_{2}=$ $0.09, \sigma_{1}=0.1, \sigma_{2}=0.3$.

\section{Acknowledgments}

This work was supported by National Natural Science Foundation of China (71501099) and Natural Science Foundation for Youths of Jiangsu of China (BK20150725). 


\section{References}

[1] H. Johnson and R. Stulz, "The pricing of options with default risk," The Journal of Finance, vol. 42, no. 2, pp. 267-280, 1987.

[2] P. Klein, "Pricing black-scholes options with correlated credit risk," Journal of Banking \& Finance, vol. 20, no. 7, pp. 1211-1229, 1996.

[3] J. Hull and A. White, "The impact of default risk on the prices of options and other derivative securities," Journal of Banking \& Finance, vol. 19, no. 2, pp. 299-322, 1995.

[4] R. A. Jarrow and S. M. Turnbull, "Pricing derivatives on financial securities subject to credit risk," Journal of Finance, vol. 50, pp. 53-85, 1995.

[5] P. Klein and M. Inglis, "Pricing vulnerable European options when the option's payoff can increase the risk of financial distress," Journal of Banking \& Finance, vol. 25, no. 5, pp. 9931012, 2001.

[6] M.-W. Hung and Y.-H. Liu, "Pricing vulnerable options in incomplete markets," Journal of Futures Markets, vol. 25, no. 2, pp. 135-170, 2005.

[7] L.-F. Chang and M.-W. Huang, "Valuation of vulnerable american options with correlated credit risk," Review of Derivatives Research, vol. 9, no. 2, pp. 137-165, 2006.

[8] P. Klein and J. Yang, "Vulnerable American options," Managerial Finance, vol. 36, no. 5, pp. 414-430, 2010.

[9] R. C. Merton, "Option pricing when underlying stock returns are discontinuous," Journal of Financial Economics, vol. 3, no. 1-2, pp. 125-144, 1976.

[10] S. G. Kou, "A jump-diffusion model for option pricing," Management Science, vol. 48, no. 8, pp. 1086-1101, 2002.

[11] W. Xu, W. Xu, H. Li, and W. Xiao, "A jump-diffusion approach to modelling vulnerable option pricing," Finance Research Letters, vol. 9, no. 1, pp. 48-56, 2012.

[12] L. Tian, G. Wang, X. Wang, and Y. Wang, "Pricing vulnerable options with correlated credit risk under jump-diffusion processes," Journal of Futures Markets, vol. 34, no. 10, pp. 957-979, 2013.

[13] J. D. Hamilton, "A new approach to the economic analysis of nonstationary time series and the business cycle," Econometrica, vol. 57, no. 2, pp. 357-384, 1989.

[14] R. J. Elliott and C. J. U. Osakwe, "Option pricing for pure jump processes with Markov switching compensators," Finance and Stochastics, vol. 10, no. 2, pp. 250-275, 2006.

[15] L. Bo, Y. Wang, and X. Yang, "Markov-modulated jumpdiffusions for currency option pricing," Insurance: Mathematics \& Economics, vol. 46, no. 3, pp. 461-469, 2010.

[16] W. Wang and W. Wang, "Pricing vulnerable options under a Markov-modulated regime switching model," Communications in Statistics-Theory and Methods, vol. 39, no. 19, pp. 3421-3433, 2010.

[17] H. Niu and D. Wang, "Pricing vulnerable options with correlated jump-diffusion processes depending on various states of the economy," Quantitative Finance, vol. 16, no. 7, pp. 1129-1145, 2016.

[18] R. J. Elliott, L. Aggoun, and J. B. Moore, Hidden Markov Models: Estimation and Control, Springer, NY, USA, 1994.

[19] H. U. Gerber and E. S. W. Shiu, "Option pricing by esscher transforms (with discussions)," Transactions of the Society of Actuaries, vol. 46, pp. 99-191, 1994.

[20] R. J. Elliott, L. Chan, and T. K. Siu, "Option pricing and Esscher transform under regime switching," Annals of Finance, vol. 1, no. 4, pp. 423-432, 2005.
[21] J. Buffington and R. J. Elliott, "Regime switching and European options," in Stochastic Theory and Control -Proceedings of a Workshop held in Lawrence, Kansas, vol. 280 of Lect. Notes Control Inf. Sci., pp. 73-82, Springer, Germany, 2002. 


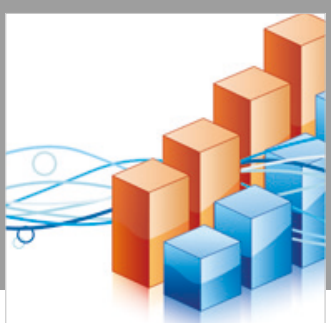

Advances in

Operations Research

\section{-n-m}
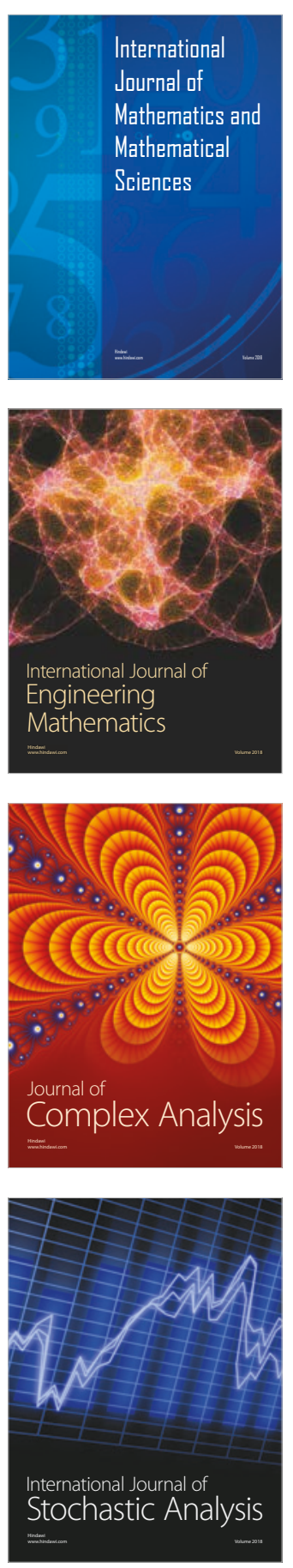
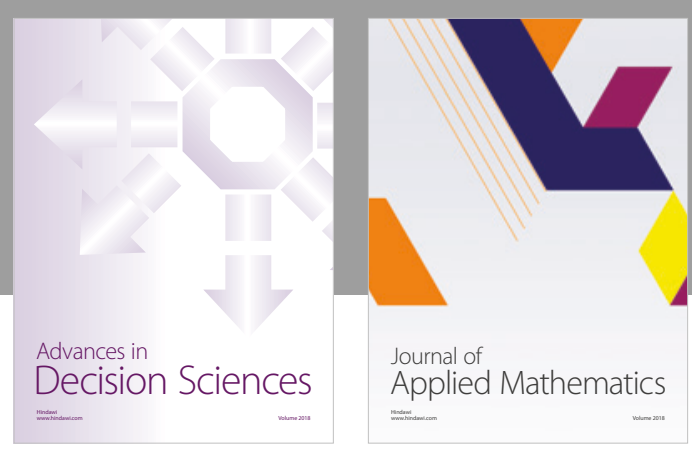

Journal of

Applied Mathematics
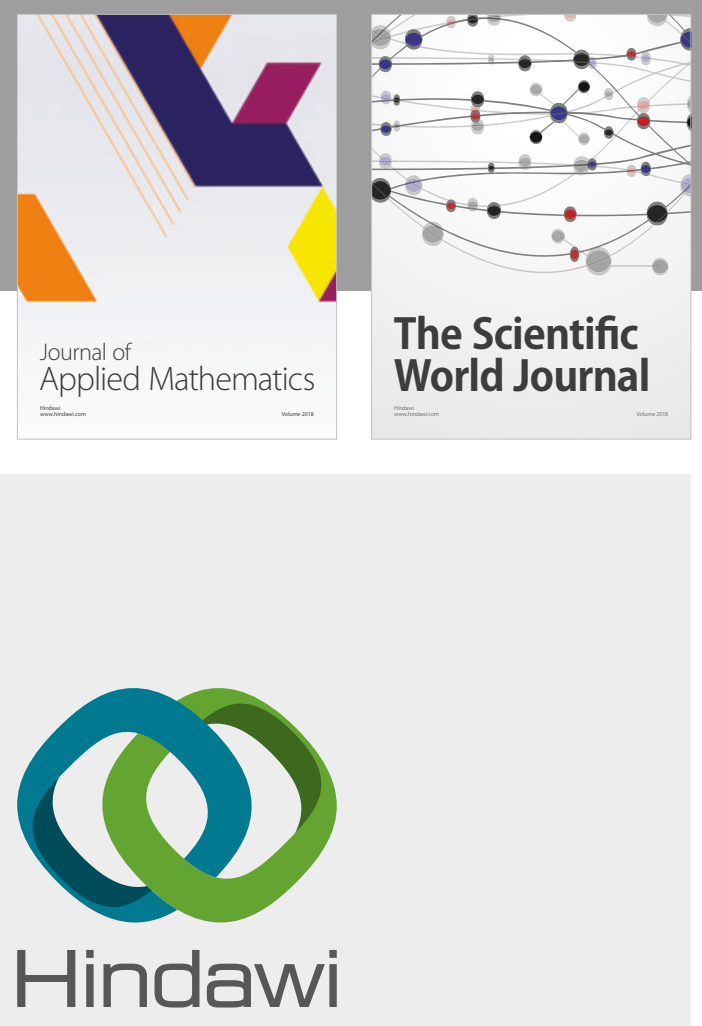

Submit your manuscripts at

www.hindawi.com



Advances in
Numerical Analysis
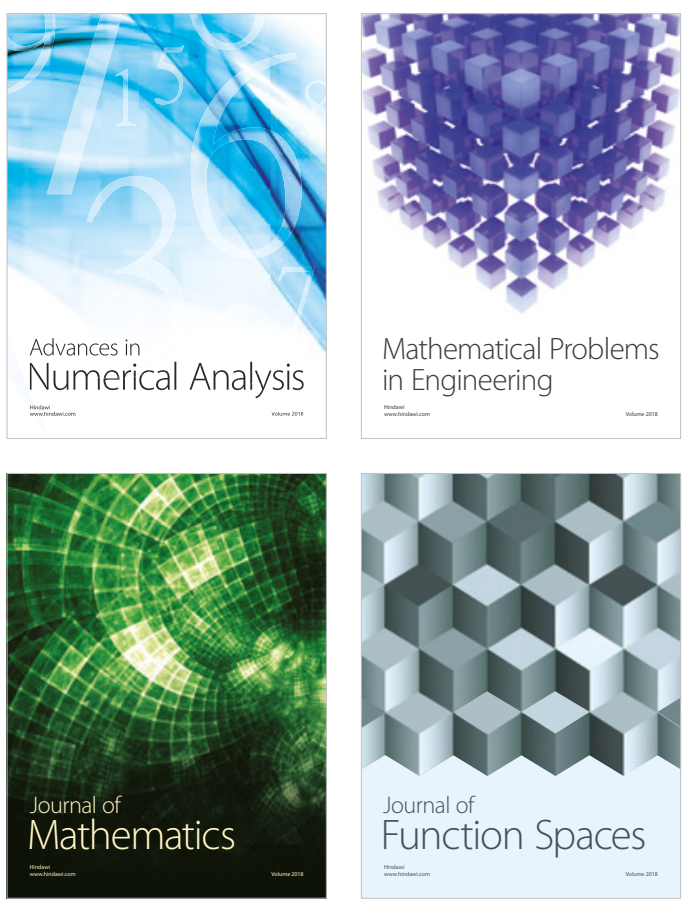

Mathematical Problems in Engineering

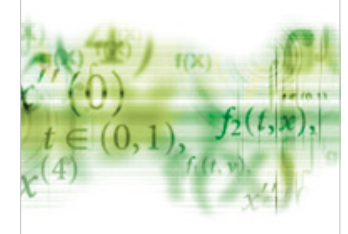

International Journal of

Differential Equations

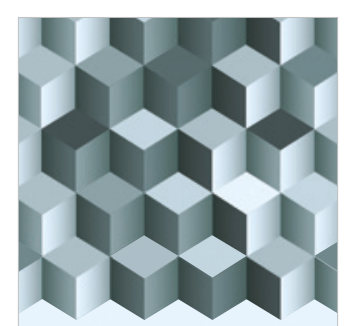

Journal of

Function Spaces

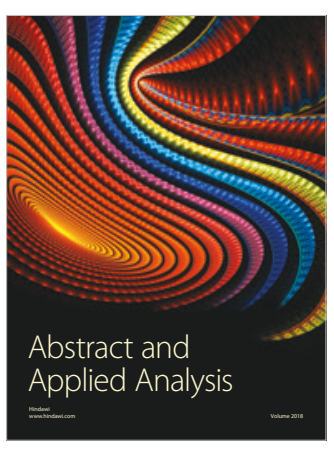

The Scientific

World Journal

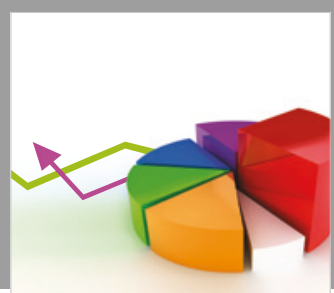

Journal of

Probability and Statistics
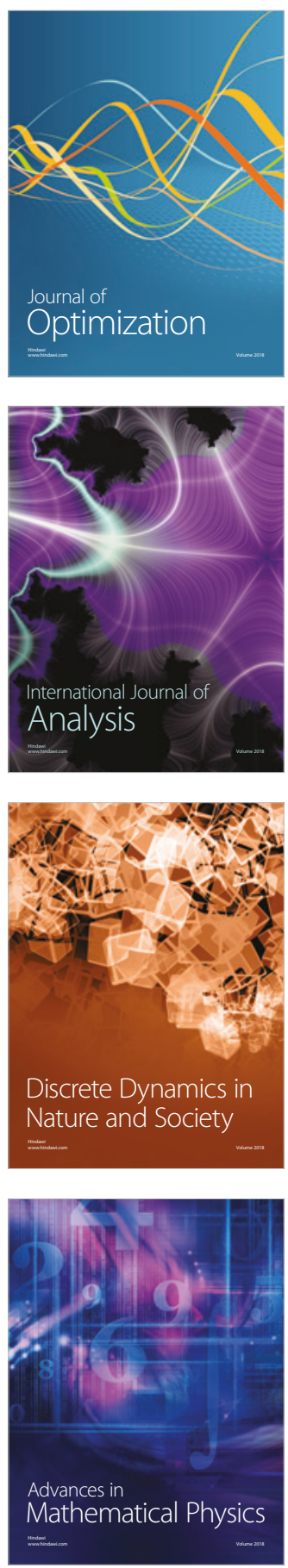